\title{
Monopoles for Gravitation and for Higher Spin Fields
}

\author{
Claudio Bunster \\ Centro de Estudios Científicos (CECS), Valdivia, Chile. \\ Sandrine Cnockaert \\ Physique Théorique et Mathématique, Université Libre de Bruxelles 8 International Solvay Institutes, \\ ULB Campus Plaine C.P. 231, B-1050 Bruxelles, Belgium. \\ Marc Henneaux \\ Physique Théorique et Mathématique, Université Libre de Bruxelles 85 International Solvay Institutes, \\ ULB Campus Plaine C.P. 231, B-1050 Bruxelles, Belgium, and \\ Centro de Estudios Científicos (CECS), Valdivia, Chile. \\ Rubén Portugues
Centro de Estudios Científicos (CECS), Valdivia, Chile.
}

\begin{abstract}
We consider massless higher spin gauge theories with both electric and magnetic sources, with a special emphasis on the spin two case. We write the equations of motion at the linear level (with conserved external sources) and introduce Dirac strings so as to derive the equations from a variational principle. We then derive a quantization condition that generalizes the familiar Dirac quantization condition, and which involves the conserved charges associated with the asymptotic symmetries for higher spins. Next we discuss briefly how the result extends to the non linear theory. This is done in the context of gravitation, where the Taub-NUT solution provides the exact solution of the field equations with both types of sources. We rederive, in analogy with electromagnetism, the quantization condition from the quantization of the angular momentum. We also observe that the Taub-NUT metric is asymptotically flat at spatial infinity in the sense of Regge and Teitelboim (including their parity conditions). It follows, in particular, that one can consistently consider in the variational principle configurations with different electric and magnetic masses.
\end{abstract}

\section{INTRODUCTION}

The idea of electric-magnetic duality has resiliently resisted developments and appears to be here to stay. Originally explored by Dirac for electromagnetism [1,2], it was later analyzed in the context of non-abelian gauge theories in $[3-5]$. It was more recently generalized to extended objects and $p$-form gauge fields in $[6,7]$. A fascinating implication of electric-magnetic duality is the charge quantization condition. This condition is antisymmetric for p-dyons of even spatial dimension $\mathrm{p}$, and symmetric for odd $\mathrm{p}$ [8-10].

Fields with spin higher than that of the graviton were discussed some time ago by Fronsdal [11] who gave the action integral for the free theories (see also [12]). Interest in these higher spin fields has been renewed since they appear in the spectrum of string theory. In particular, duality for free higher spins has recently been the focus of many papers [13-18]. Conserved, external, electric-type sources can easily be coupled to the higher spin fields but the problem of constructing consistent self-interactions is still incompletely understood beyond spin 2 (for instance, the action principle is not known even though non linear equations have been constructed in the remarkable work [19]). It has been argued that self-interactions can be brought in but only among an infinite tower of fields [19].

Since duality can be defined for higher spins and since electric sources can be included, one might wonder whether magnetic sources can be considered as well. Our paper solves positively this question for all spins at the linearized level and provides additional insight in the full non linear theory for spin 2.

We show that conserved external sources of both types can be coupled to any given higher (integer) spin field within the context of the linear theory. The presence of magnetic sources requires the introduction of Dirac strings, as in the spin 1 case. To preserve manifest covariance, the location of the string must be left arbitrary and is, in fact, unobservable classically. The requirement that the Dirac string is unobservable quantum-mechanically forces a quantization condition of the form

$$
\frac{1}{2 \pi \hbar} Q_{\gamma_{1} \cdots \gamma_{s-1}}(v) P^{\gamma_{1} \cdots \gamma_{s-1}}(u) \in \mathbb{Z}
$$

Here, the symmetric tensor $P^{\gamma_{1} \cdots \gamma_{s-1}}(u)$ is the conserved electric charge associated with the asymptotic symmetries of the spin s field, while $Q_{\gamma_{1} \cdots \gamma_{s-1}}(v)$ is the corresponding "topological" magnetic charge. For s $=1$, the asymptotic symmetries are internal symmetries and, actually, just constant phase transformations. The conserved charge $P$ is the 
electric charge $q$ while $Q$ is the magnetic charge $g$, yielding the familiar Dirac quantization condition for the product of electric and magnetic charges. For $\mathrm{s}=2$ the conserved charges have a spacetime index and the quantization condition reads (after rescaling the conserved quantities so they have dimensions of mass)

$$
\frac{4 G P_{\gamma} Q^{\gamma}}{\hbar} \in \mathbb{Z}
$$

The quantity $P_{\gamma}$ is the "electric" 4-momentum associated with constant linearized diffeomorphisms (translations) while $Q_{\gamma}$ is the corresponding magnetic 4-momentum. For a point particle source, $P_{\gamma}=M u_{\gamma}$ where $M$ is the "electric" mass and $u_{\gamma}$ the 4-velocity of the electric source. Similarly, $Q_{\gamma}=N v_{\gamma}$ where $N$ is the "magnetic" mass and $v_{\gamma}$ the 4 -velocity of the magnetic source.

All this is just a generalization of the familiar spin 1 case, although the explicit introduction of the Dirac string is more intricate for higher spins because the gauge invariance is then more delicate to control. Indeed, there is no gauge invariant object that involves first derivatives of the fields only ( $s>1)$. Hence, the Lagrangian is not strictly gauge invariant, contrary to what happens for electromagnetism, but is gauge invariant only up to a total derivative.

A serious limitation of the linear theory for $\mathrm{s}>1$ is that the sources must follow straight lines. This follows from the strict conservation laws implied by the field equations, which are much more stringent for $\mathrm{s}>1$ than they are for $\mathrm{s}=$ 1. Thus the sources must be treated as externally given and cannot be freely varied in the variational principle. One cannot study the back reaction of the spin s field on the sources without introducing self-interactions. This problem occurs already for the spin 2 case and has nothing to do with the introduction of magnetic sources.

We have not investigated the backreaction problem for general spins $\mathrm{s}>2$ since the non linear theory is still a subject of investigation even in the absence of sources. We have focused instead on the spin 2 case, for which the non linear theory is given by the Einstein theory of gravity. The remarkable Taub-NUT solution [20], which represents the vacuum exterior field of a gravitational dyon, indicates that Einstein's theory can support both electric and magnetic masses. The remaining part of our paper is devoted to a further discussion of the Taub-NUT solution, including an analysis of its asymptotic properties. We provide new insight on the quantization condition (I.2) through the analysis of the angular momentum stored in the gravitational field of a pair of electric and magnetic masses placed at different locations.

Our paper is organized as follows. In section II, we consider in detail the spin 2 case with point particle electric and magnetic sources. We introduce Dirac strings and derive the quantization condition. We then extend the formalism to higher spins (section III), again with point particle sources. In section IV we consider the Taub-NUT solution and discuss the quantization condition in the non linear context. After a brief concluding section, the article ends with three appendices where technical aspects for spins greater than 2 that were not included in section III are dealt with (Appendices A, B and C). Finally some aspects of the angular momentum of an electric test particle in the field of a monopole, which turn out to be relevant to the discussion of the Taub-NUT metric are covered in Appendix D.

\section{LINEARIZED GRAVITY WITH ELECTRIC AND MAGNETIC MASSES}

\section{A. Electric and magnetic sources}

The equations of motion for linearized gravity coupled to both electric and magnetic sources are naturally written in terms of the linearized Riemann tensor $R_{\alpha \beta \lambda \mu}$, hereafter just called "Riemann tensor" for simplicity. This is the physical, gauge-invariant, object, analogous to the field strength $F_{\mu \nu}$ of electromagnetism. How to introduce the "potential", i.e., the symmetric spin 2 field $h_{\mu \nu}=h_{\nu \mu}$ will be discussed below. The dual to the Riemann tensor is defined as

$$
S_{\alpha \beta \lambda \mu}=-\frac{1}{2} \epsilon_{\alpha \beta \gamma \delta} R_{\lambda \mu}^{\gamma \delta} .
$$

We denote the "electric" energy-momentum tensor by $T^{\mu \nu}$ and the "magnetic" energy-momentum tensor by $\Theta^{\mu \nu}$. These are both symmetric and conserved, $T^{\mu \nu}=T^{\nu \mu}, \Theta^{\mu \nu}=\Theta^{\nu \mu}, T^{\mu \nu}{ }_{\nu}=0, \Theta^{\mu \nu}{ }_{\nu}=0$. It is also useful to define $\bar{T}^{\mu \nu}=T^{\mu \nu}-\frac{1}{2} \eta^{\mu \nu} T, \bar{\Theta}^{\mu \nu}=\Theta^{\mu \nu}-\frac{1}{2} \eta^{\mu \nu} \Theta$ where $T$ and $\Theta$ are the traces. We assume that $T^{\mu \nu}$ and $\Theta^{\mu \nu}$ have the units of an energy density. We set $c=1$ but keep $G$.

The form of the equations in the presence of both types of sources is fixed by: (i) requiring duality invariance with respect to the $S O(2)$-rotations of the curvatures and the sources [17],

$$
\begin{aligned}
R_{\alpha \beta \lambda \mu}^{\prime} & =\cos \alpha R_{\alpha \beta \lambda \mu}+\sin \alpha S_{\alpha \beta \lambda \mu}, & S_{\alpha \beta \lambda \mu}^{\prime} & =-\sin \alpha R_{\alpha \beta \lambda \mu}+\cos \alpha S_{\alpha \beta \lambda \mu}, \\
T_{\alpha \beta}^{\prime} & =\cos \alpha T_{\alpha \beta}+\sin \alpha \Theta_{\alpha \beta}, & \Theta_{\alpha \beta}^{\prime} & =-\sin \alpha T_{\alpha \beta}+\cos \alpha \Theta_{\alpha \beta},
\end{aligned}
$$


and, (ii) using the known form of the equations in the presence of electric masses only. One finds explicitly the following:

- The Riemann tensor is antisymmetric in the first two indices and the last two indices, but in general is not symmetric for the exchange of the pairs, i.e., $R_{\alpha \beta \lambda \mu}=-R_{\beta \alpha \lambda \mu}, R_{\alpha \beta \lambda \mu}=-R_{\alpha \beta \mu \lambda}$ with $R_{\alpha \beta \lambda \mu} \neq R_{\lambda \mu \alpha \beta}$ (in the presence of magnetic sources).

- In the presence of magnetic sources the cyclic identity is [33]

$$
R_{\alpha \beta \lambda \mu}+R_{\beta \lambda \alpha \mu}+R_{\lambda \alpha \beta \mu}=8 \pi G \epsilon_{\alpha \beta \lambda \nu} \bar{\Theta}_{\mu}^{\nu} .
$$

This enables one to relate $R_{\alpha \beta \lambda \mu}$ to $R_{\lambda \mu \alpha \beta}$ through

$$
R_{\alpha \beta \gamma \delta}-R_{\gamma \delta \alpha \beta}=4 \pi G\left(\epsilon_{\alpha \beta \gamma \lambda} \bar{\Theta}_{\delta}^{\lambda}-\epsilon_{\alpha \beta \delta \lambda} \bar{\Theta}_{\gamma}^{\lambda}+\epsilon_{\beta \gamma \delta \lambda} \bar{\Theta}_{\alpha}^{\lambda}-\epsilon_{\alpha \gamma \delta \lambda} \bar{\Theta}_{\beta}^{\lambda}\right) .
$$

It follows that the Ricci tensor is symmetric, $R_{\lambda \mu}=R_{\mu \lambda}$. The Einstein tensor $G_{\lambda \mu}=R_{\lambda \mu}-(1 / 2) \eta_{\lambda \mu} R$ is then also symmetric.

- The Bianchi identity is

$$
\partial_{\epsilon} R_{\alpha \beta \gamma \delta}+\partial_{\alpha} R_{\beta \epsilon \gamma \delta}+\partial_{\beta} R_{\epsilon \alpha \gamma \delta}=8 \pi G \epsilon_{\epsilon \alpha \beta \rho}\left(\partial_{\gamma} \bar{\Theta}_{\delta}^{\rho}-\partial_{\delta} \bar{\Theta}_{\gamma}^{\rho}\right) .
$$

Although there is now a right-hand side in the Bianchi identity, the contracted Bianchi identities are easily verified to be unaffected and still read

$$
G_{, \mu}^{\lambda \mu}=0
$$

- The Einstein equations are

$$
G^{\lambda \mu}=8 \pi G T^{\lambda \mu}
$$

or equivalently, $R^{\lambda \mu}=8 \pi G \bar{T}^{\lambda \mu}$, and force exact conservation of the sources because of the contracted Bianchi identity, as in the absence of magnetic mass.

The equations are completely symmetric under duality. Indeed, one easily checks that one gets the same equations for the dual curvature $S_{\alpha \beta \lambda \mu}$ with the roles of the electric and magnetic energy-momentum tensors exchanged. In the course of the verification of this property, the equation

$$
\partial^{\mu} R_{\mu \rho \gamma \delta}=8 \pi G\left(\partial_{\gamma} \bar{T}_{\rho \delta}-\partial_{\delta} \bar{T}_{\rho \gamma}\right)
$$

which follows from (II.2), (II.3) and the conservation of $\Theta^{\mu \nu}$ are useful. Furthermore, in the absence of magnetic sources, one recovers the equations of the standard linearized Einstein theory since the cyclic and Bianchi identities have no source term in their right hand sides.

The formalism can be extended to include a cosmological constant $\Lambda$. The relevant curvature is then the MacDowellMansouri curvature [21] linearized around (anti) de Sitter space [18]. In terms of this tensor, the equations (II.1), (II.3) and (II.5) take the same form, with ordinary derivatives replaced by covariant derivatives with respect to the (anti) de Sitter background.

\section{B. Decomposition of the Riemann tensor - Spin 2 field}

We exhibit a variational principle from which the equations of motion follow. To that end, we first need to indicate how to introduce the spin 2 field $h_{\mu \nu}$.

Because there are right-hand sides in the cyclic and Bianchi identities, the Riemann tensor is not directly derived from a potential $h_{\mu \nu}$. To introduce $h_{\mu \nu}$, we split $R_{\lambda \mu \alpha \beta}$ into a part that obeys the cyclic and Bianchi identities and a part that is fixed by the magnetic energy-momentum tensor. Let $\Phi_{\gamma}^{\alpha \beta}$ be such that

$$
\partial_{\alpha} \Phi_{\gamma}^{\alpha \beta}=16 \pi G \Theta_{\gamma}^{\beta}, \quad \Phi_{\gamma}^{\alpha \beta}=-\Phi_{\gamma}^{\beta \alpha} .
$$

We shall construct $\Phi_{\gamma}^{\alpha \beta}$ in terms of $\Theta_{\gamma}^{\beta}$ and Dirac strings below. We set

$$
R_{\lambda \mu \alpha \beta}=r_{\lambda \mu \alpha \beta}+\frac{1}{4} \epsilon_{\lambda \mu \rho \sigma}\left(\partial_{\alpha} \bar{\Phi}_{\beta}^{\rho \sigma}-\partial_{\beta} \bar{\Phi}_{\alpha}^{\rho \sigma}\right)
$$


with

$$
\bar{\Phi}_{\alpha}^{\rho \sigma}=\Phi_{\alpha}^{\rho \sigma}+\frac{1}{2}\left(\delta_{\alpha}^{\rho} \Phi^{\sigma}-\delta_{\alpha}^{\sigma} \Phi^{\rho}\right), \quad \Phi^{\rho} \equiv \Phi_{\sigma}^{\rho \sigma} .
$$

Using $\partial_{\alpha} \bar{\Phi}_{\gamma}^{\alpha \beta}=16 \pi G \bar{\Theta}_{\gamma}^{\beta}-\partial_{\gamma} \bar{\Phi}^{\beta}, \bar{\Phi}^{\beta}=-\frac{1}{2} \Phi^{\beta}$, one easily verifies that the cyclic and Bianchi identities take the standard form when written in terms of $r_{\alpha \beta \lambda \mu}$, namely,

$$
r_{\alpha \beta \lambda \mu}+r_{\beta \lambda \alpha \mu}+r_{\lambda \alpha \beta \mu}=0, \quad \partial_{\epsilon} r_{\alpha \beta \gamma \delta}+\partial_{\alpha} r_{\beta \epsilon \gamma \delta}+\partial_{\beta} r_{\epsilon \alpha \gamma \delta}=0 .
$$

Hence, there exists a symmetric tensor $h_{\mu \nu}$ such that $r_{\alpha \beta \lambda \mu}=-2 \partial_{[\beta} h_{\alpha][\lambda, \mu]}$.

If one sets $y_{\gamma}^{\lambda \mu}=\epsilon^{\lambda \mu \rho \sigma} \partial_{\rho} h_{\sigma \gamma}=-y_{\gamma}^{\mu \lambda}$, one may rewrite the curvature as

$$
R_{\lambda \mu \alpha \beta}=\frac{1}{4} \epsilon_{\lambda \mu \rho \sigma}\left(\partial_{\alpha} \bar{Y}_{\beta}^{\rho \sigma}-\partial_{\beta} \bar{Y}_{\alpha}^{\rho \sigma}\right)
$$

with

$$
Y_{\beta}^{\rho \sigma}=y_{\beta}^{\rho \sigma}+\Phi_{\beta}^{\rho \sigma}=-Y_{\beta}^{\sigma \rho}, \quad \bar{Y}_{\alpha}^{\rho \sigma}=Y_{\alpha}^{\rho \sigma}+\frac{1}{2}\left(\delta_{\alpha}^{\rho} Y^{\sigma}-\delta_{\alpha}^{\sigma} Y^{\rho}\right), \quad Y^{\rho} \equiv Y_{\sigma}^{\rho \sigma},
$$

(note that $\bar{y}_{\alpha}^{\rho \sigma}=y_{\alpha}^{\rho \sigma}$ and that $\partial_{\rho} y_{\alpha}^{\rho \sigma}=0$ ).

\section{Dirac string}

We consider point particle sources. The particles must be forced to follow straight lines because of the conservation equations $T_{, \nu}^{\mu \nu}=0$ and $\Theta^{\mu \nu}{ }_{, \nu}=0$. If $u^{\mu}$ is the 4-velocity of the electric source and $v^{\mu}$ the 4-velocity of the magnetic source, one has

$$
T^{\mu}{ }_{\nu}=M u_{\nu} \int d \lambda \delta^{(4)}(x-z(\lambda)) \dot{z}^{\mu}, \quad \Theta^{\mu}{ }_{\nu}=N v_{\nu} \int d \lambda \delta^{(4)}(x-\bar{z}(\lambda)) \dot{\bar{z}}^{\mu},
$$

where $z^{\mu}(\lambda)$ and $\left.\bar{z}^{\mu}(\lambda)\right)$ are the worldlines of the electric and magnetic sources respectively, e.g. $u^{\mu}=d z^{\mu} / d s$. Performing the integral, one finds

$$
T^{\mu \nu}=\frac{u^{\mu} u^{\nu}}{u^{0}} \delta^{(3)}\left(\vec{x}-\vec{z}\left(x^{0}\right)\right), \quad \Theta^{\mu \nu}=\frac{v^{\mu} v^{\nu}}{v^{0}} \delta^{(3)}\left(\vec{x}-\vec{z}\left(x^{0}\right)\right) .
$$

The tensor $\Phi_{\gamma}^{\alpha \beta}$ introduced in (II.6) can be constructed à la Dirac [2], by attaching a Dirac string $y^{\mu}(\lambda, \sigma)$ to the magnetic source, $y^{\mu}(\lambda, 0)=\bar{z}^{\mu}(\lambda)$. One has explicitly

$$
\Phi_{\gamma}^{\alpha \beta}=16 \pi G N v_{\gamma} \int d \lambda d \sigma\left(y^{\alpha} \dot{y}^{\beta}-\dot{y}^{\alpha} y^{\prime \beta}\right) \delta^{(4)}(x-y(\lambda, \sigma)),
$$

where

$$
\dot{y}^{\alpha}=\frac{\partial y^{\alpha}}{\partial \lambda}, \quad y^{\prime \alpha}=\frac{\partial y^{\alpha}}{\partial \sigma} .
$$

One verifies exactly as for electromagnetism that the divergence of $\Phi_{\gamma}^{\alpha \beta}$ is equal to the magnetic energy-momentum tensor (up to the factor $16 \pi G$ ). What plays the role of the magnetic charge $g$ in electromagnetism is now the conserved product $N v_{\mu}$ of the magnetic mass of the source by its 4 -velocity. This is the magnetic 4-momentum.

\section{Variational principle}

\section{Action}

When the curvature is expressed in terms of $h_{\mu \nu}$ as in (II.8), the expressions (II.1) and (II.3) are identically fulfilled and the relations (II.5) become equations of motion for $h_{\mu \nu}$. These equations can be derived from a variational principle which we now describe. 
The action that yields (II.5) is

$$
S\left[h_{\mu \nu}(x), y^{\mu}(\lambda, \sigma)\right]=\frac{1}{16 \pi G} \int \frac{1}{4}\left(\bar{Y}_{\alpha \beta \gamma} \bar{Y}^{\alpha \gamma \beta}-\bar{Y}_{\alpha} \bar{Y}^{\alpha}\right) d^{4} x+\frac{1}{2} \int h_{\mu \nu} T^{\mu \nu} d^{4} x .
$$

One varies the fields $h_{\mu \nu}$ and the coordinates $y^{\mu}$ of the string (with the condition that it remains attached to the magnetic source), but not the trajectories of the sources, which are fixed because of the conservation laws $\partial_{\mu} T^{\mu \nu}=0$ and $\partial_{\mu} \Theta^{\mu \nu}=0$. This is a well known limitation of the linearized theory, present already in the pure electric case. To treat the sources as dynamical, one needs to go beyond the linear theory.

If there is no magnetic source, the first term in the action reduces to

$$
S^{P F}=\frac{1}{16 \pi G} \int \frac{1}{4}\left(-\partial_{\lambda} h_{\alpha \beta} \partial^{\lambda} h^{\alpha \beta}+2 \partial_{\lambda} h^{\lambda \alpha} \partial^{\mu} h_{\mu \alpha}-2 \partial^{\lambda} h \partial_{\mu} h^{\mu \lambda}+\partial_{\lambda} h \partial^{\lambda} h\right),
$$

which is the Pauli-Fierz action, whose variation with respect to $h_{\alpha \beta}$ gives $-\frac{1}{16 \pi G}$ times the linearized Einstein tensor $G^{\alpha \beta}$. It is straightforward to verify that the variation of the first term in the action with respect to $h_{\alpha \beta}$ still gives $-\frac{1}{16 \pi G}$ times the linearized Einstein tensor $G^{\alpha \beta}$ with correct $\Phi^{\mu \nu}{ }_{\alpha}$ contributions even in the presence of magnetic sources. So, the equations of motion that follow from (II.12) when one varies the gravitational field are the Einstein equations (II.5).

Extremization with respect to the string coordinates does not bring in new conditions provided that the Dirac string does not go through an electric source (Dirac veto).

The action (II.12) was obtained by using the analysis of source-free linearized gravity in terms of two independent fields given in [16], which enables one to go from the electric to the magnetic formulations and vice-versa, by elimination of magnetic or electric variables. As one knows how to introduce electric sources in the electric formulation, through standard minimal coupling, one can find how these sources appear in the magnetic formulation by eliminating the electric variables and keeping the magnetic potentials. So, one can determine how to introduce electric poles in the magnetic formulation, or, what is equivalent, magnetic poles in the electric formulation.

\section{Gauge invariances}

\section{Diffeomorphism invariance}

The action (II.12) is invariant under linearized diffeomorphisms and under displacements of the Dirac string (accompanied by appropriate transformations of the spin 2 field). The easiest way to show this is to observe that the first term in the action (II.12) is invariant if one shifts $Y_{\alpha}^{\mu \nu}$ according to

$$
Y_{\alpha}^{\mu \nu} \rightarrow Y_{\alpha}^{\mu \nu}+\delta_{\alpha}^{\mu} \partial_{\rho} z^{\nu \rho}-\delta_{\alpha}^{\nu} \partial_{\rho} z^{\mu \rho}+\partial_{\alpha} z^{\mu \nu},
$$

where $z^{\mu \nu}=-z^{\nu \mu}$ is arbitrary. This is most directly verified by noting that under (II.13), the tensor $\bar{Y}^{\mu \nu}{ }_{\alpha}$ defined in (II.9) transforms simply as

$$
\bar{Y}_{\alpha}^{\mu \nu} \rightarrow \bar{Y}_{\alpha}^{\mu \nu}+\partial_{\alpha} z^{\mu \nu}
$$

and this leaves invariant the first term in (II.12) up to a total derivative. Note that the Riemann tensor (II.8) is strictly invariant. The transformation (II.13) can be conveniently rewritten as

$$
Y_{\alpha}^{\mu \nu} \rightarrow Y_{\alpha}^{\mu \nu}+\epsilon^{\mu \nu \rho \sigma} \partial_{\rho} a_{\sigma \alpha},
$$

where $a_{\sigma \alpha}=-a_{\alpha \sigma}$ is given by $a_{\sigma \alpha}=\frac{1}{2} \epsilon_{\sigma \alpha \beta \gamma} z^{\beta \gamma}$.

A (linearized) diffeomorphism

$$
h_{\mu \nu} \rightarrow h_{\mu \nu}+\partial_{\mu} \xi_{\nu}+\partial_{\nu} \xi_{\mu}
$$

(with the string coordinates unaffected) modifies $Y^{\mu \nu}{ }_{\alpha}$ as in (II.15) with $a_{\sigma \alpha}=\partial_{\alpha} \xi_{\sigma}-\partial_{\sigma} \xi_{\alpha}$ (note that the term $\partial_{\sigma} \xi_{\alpha}$ in $a_{\sigma \alpha}$ does not contribute because $\left.\partial_{[\rho} \partial_{\sigma]} \xi_{\alpha}=0\right)$. Hence, the first term in the action (II.12) is invariant under diffeomorphisms. The minimal coupling term is also invariant because the energy-momentum tensor is conserved. It follows that the complete action (II.12) is invariant under diffeomorphisms. 


\section{Displacements of the Dirac string}

An arbitrary displacement of the Dirac string,

$$
y^{\alpha}(\tau, \sigma) \rightarrow y^{\alpha}(\tau, \sigma)+\delta y^{\alpha}(\tau, \sigma)
$$

also modifies $Y_{\alpha}^{\mu \nu}$ as in (II.15) provided one transforms simultaneously the spin 2 field $h_{\mu \nu}$ appropriately. Indeed, under the displacement (II.17) of the Dirac string, the quantity $\Phi_{\alpha}^{\mu \nu}$ changes as $\Phi^{\mu \nu}{ }_{\alpha} \rightarrow \Phi^{\mu \nu}{ }_{\alpha}+k^{\mu \nu}{ }_{\alpha}$ where $k^{\mu \nu}{ }_{\alpha}$ can be computed from $\delta y^{\alpha}(\tau, \sigma)$ through (II.11) and has support on the old and new string locations. Its explicit expression will not be needed. What will be needed is that it fulfills

$$
\partial_{\mu} k_{\alpha}^{\mu \nu}=0
$$

because the magnetic energy-momentum tensor is not modified under a displacement of the Dirac string. The field $Y^{\mu \nu}{ }_{\alpha}$ changes then as

$$
Y_{\alpha}^{\mu \nu} \rightarrow Y_{\alpha}^{\mu \nu}+\epsilon^{\mu \nu \rho \sigma} \partial_{\rho} \delta h_{\sigma \alpha}+k_{\alpha}^{\mu \nu}
$$

where $\delta h_{\sigma \alpha}$ is the sought after variation of $h_{\sigma \alpha}$. By using (II.18), one may rewrite the last term in (II.19) as $\partial_{\rho} t^{\mu \nu \rho}{ }_{\alpha}$ for some $t^{\mu \nu \rho}{ }_{\alpha}=t^{[\mu \nu \rho]}$. Again, we shall not need an explicit expression for $t^{\mu \nu \rho}{ }_{\alpha}$, but only the fact that because $k^{\mu \nu}{ }_{\alpha}$ has support on the string locations, which do not go through the electric sources (Dirac veto), one may choose $t^{\mu \nu \rho}{ }_{\alpha}$ to vanish on the electric sources as well. In fact, one may take $t^{\mu \nu \rho}{ }_{\alpha}$ to be non-vanishing only on a membrane supported by the string. Decomposing $t^{\mu \nu \rho}{ }_{\alpha}$ as $t^{\mu \nu \rho}{ }_{\alpha}=\epsilon^{\mu \nu \rho \sigma}\left(s_{\sigma \alpha}+a_{\sigma \alpha}\right), s_{\sigma \alpha}=s_{(\sigma \alpha)}, a_{\sigma \alpha}=a_{[\sigma \alpha]}$ and taking $h_{\sigma \alpha}$ to transform as $h_{\sigma \alpha} \rightarrow h_{\sigma \alpha}-s_{\sigma \alpha}$ one sees from (II.19) that the variation of $Y^{\mu \nu}{ }_{\alpha}$ takes indeed the form (II.15). Thus the first term in the action is invariant. The minimal coupling term is also invariant because the support of the variation of the spin 2 field does not contain the electric worldlines.

One can also observe that the variation $\delta r_{\alpha \beta \rho \sigma}$ vanishes outside the original and displaced string locations. This implies $\delta h_{\alpha \beta}=\partial_{\alpha} \xi_{\beta}+\partial_{\beta} \xi_{\alpha}$ except on the location of both strings, where $\xi_{\alpha}$ induces a delta function contribution on the string ("singular gauge transformation"). The explicit expressions will not be given here.

\section{Identities}

The identities which follow from the invariance (II.13), or (II.15), of the first term

$$
\mathcal{L}=\frac{1}{64 \pi G}\left(\bar{Y}_{\alpha \beta \gamma} \bar{Y}^{\alpha \gamma \beta}-\bar{Y}_{\alpha} \bar{Y}^{\alpha}\right)
$$

in the action may be written as

$$
\partial_{\rho}\left(\frac{\partial \mathcal{L}}{\partial Y_{\gamma}^{\alpha \beta}}\right) \epsilon^{\alpha \beta \rho \sigma}=\partial_{\rho}\left(\frac{\partial \mathcal{L}}{\partial Y_{\sigma}^{\alpha \beta}}\right) \epsilon^{\alpha \beta \rho \gamma}
$$

They imply that

$$
\begin{aligned}
-\frac{1}{16 \pi G} G^{\alpha \beta}=\frac{\delta \mathcal{L}}{\delta h_{\alpha \beta}} & =-\partial_{\rho}\left(\frac{\partial \mathcal{L}}{\partial Y^{\mu \nu}}\right) \epsilon^{\mu \nu \rho \alpha} \\
& =-\partial_{\rho}\left(\frac{\partial \mathcal{L}}{\partial Y_{\alpha}^{\mu \nu}}\right) \epsilon^{\mu \nu \rho \beta},
\end{aligned}
$$

from which the contracted Bianchi identities are easily seen to indeed hold.

The expression (II.8) of the Riemann tensor in terms of $\bar{Y}^{\mu \nu}{ }_{\alpha}$ makes it clear that it is invariant under (II.14) and thus, invariant under both diffeomorphisms and displacements of the Dirac string.

\section{E. Quantization condition}

Because of the gauge invariances just described, the Dirac string is classically unobservable. In the Hamiltonian formalism, this translates itself into the existence of first-class constraints expressing the momenta conjugate to the string coordinates in terms of the remaining variables. Demanding that the string remains unobservable in the quantum theory imposes a quantization condition on the charges, which we now derive. The argument follows closely that of Dirac in the electromagnetic case [2]. 
Working for simplicity in the gauge $y^{0}=\lambda$ (which eliminates $y^{0}$ as an independent variable), one finds the constraints

$$
\pi_{m}=-32 \pi G N y^{\prime n} v_{\gamma} \frac{\partial \mathcal{L}}{\partial Y_{\gamma}^{m n}}
$$

The right hand side of (II.23) generates the change of the gravitational field that accompanies the displacement of the Dirac string.

In the quantum theory, the wave functional $\psi$ must therefore fulfill

$$
\frac{\hbar}{i} \frac{\delta \psi}{\delta y^{m}(\sigma)}=-32 \pi G N y^{\prime n} v_{\gamma} \frac{\partial \mathcal{L}}{\partial Y_{\gamma}^{m n}} \psi
$$

We integrate this equation as in [2], along a path in the configuration space of the string that encloses an electric source. One finds that the variation of the phase of the wave functional is given by

$$
\Delta \Psi=-\frac{16 \pi G N v_{\gamma}}{\hbar} \int \frac{\partial \mathcal{L}}{\partial Y_{\gamma}^{m n}}\left(\dot{y}^{m} y^{\prime n}-\dot{y}^{n} y^{\prime m}\right) d \sigma d \lambda
$$

where the integral is taken on the two-dimensional surface enclosing the electric source. Using the Gauss theorem, this can be converted to a volume integral,

$$
\Delta \Psi=-\frac{16 \pi G N v_{\gamma}}{\hbar} \int d^{3} x \epsilon^{m n p} \partial_{p}\left(\frac{\partial \mathcal{L}}{\partial Y_{\gamma}^{m n}}\right) .
$$

Because $\epsilon^{m n p} \partial_{p}\left(\frac{\partial \mathcal{L}}{\partial Y_{\gamma}^{m n}}\right)=\frac{\delta \mathcal{L}}{\delta h_{0 \gamma}}$, the variation of the phase becomes, upon use of the constraint (initial value) Einstein equations $G^{0 \gamma}=8 \pi G T^{0 \gamma}$,

$$
\Delta \Psi=\frac{8 \pi G N v_{\gamma}}{\hbar} \int d^{3} x T^{0 \gamma}=\frac{8 \pi G N M v_{\gamma} u^{\gamma}}{\hbar} .
$$

For the wave functional to be single-valued, this should be a multiple of $2 \pi$. This yields the quantization condition

$$
\frac{4 N M G v_{\gamma} u^{\gamma}}{\hbar}=n, \quad n \in \mathbb{Z}
$$

Introducing the conserved charges $P^{\gamma}, Q^{\gamma}$ associated with the spin 2 theory (electric and magnetic 4-momentum), this can be rewritten as

$$
\frac{4 G P_{\gamma} Q^{\gamma}}{\hbar} \in \mathbb{Z}
$$

It is to be stressed that the quantization condition is not a condition on the electric and magnetic masses, but rather, on the electric and magnetic 4-momenta. In the rest frame of the magnetic source, the quantization condition becomes

$$
\frac{4 G E N}{\hbar} \in \mathbb{Z}
$$

where $E$ is the (electric) energy of the electric mass. Thus, it is the energy which is quantized, not the mass.

We have taken above a pure electric source and a pure magnetic pole. We could have taken dyons, one with charges $\left(P^{\gamma}, Q^{\gamma}\right)$, the other with charges $\left(\bar{P}^{\gamma}, \bar{Q}^{\gamma}\right)$. Then the quantization condition reads

$$
\frac{4 G\left(P_{\gamma} \bar{Q}^{\gamma}-\bar{P}_{\gamma} Q^{\gamma}\right)}{\hbar} \equiv \frac{4 G \epsilon_{a b} Q_{\gamma}^{a} \bar{Q}^{b \gamma}}{\hbar} \in \mathbb{Z},
$$

since the sources are pointlike (0-dyons). Here $Q_{\gamma}^{a} \equiv\left(P_{\gamma}, Q_{\gamma}\right), a, b=1,2$ and $\epsilon_{a b}$ is the $S O(2)$-invariant Levi-Civita tensor in the 2-dimensional space of the charges. 


\section{F. One-particle solutions}

\section{Electric mass}

We consider a point particle electric mass at rest at the origin of the coordinate system. The only non-vanishing component of its electric energy momentum tensor is $T^{00}\left(x^{0}, \vec{x}\right)=M \delta^{(3)}(\vec{x})$ while $\Theta^{\mu \nu}$ vanishes. There is no Dirac string since there is no magnetic mass. The metric generated by this source is static. The linearized Einstein equations are well known to imply in that case the linearized Schwarzschild solution, namely in polar coordinates

$$
h_{00}=\frac{2 G M}{r}=h_{r r}, \quad \text { other components vanish },
$$

or in Cartesian coordinates

$$
h_{00}=\frac{2 G M}{r}, \quad h_{i j}=\frac{2 G M}{r^{3}} x_{i} x_{j}, \quad \text { other components vanish. }
$$

Indeed, one then finds

$$
\begin{aligned}
R_{0 s 0 b}= & M\left(-\frac{3 x_{s} x_{b}}{r^{5}}+\frac{\delta_{s b}}{r^{3}}+\frac{4 \pi}{3} \delta_{s b} \delta(\vec{x})\right) \\
R_{0 s a b}= & 0=R_{a b 0 s}, \\
R_{p q a b}= & \left(\delta_{p a} \delta_{q b}-\delta_{p b} \delta_{q a}\right)\left(\frac{2 M}{r^{3}}+\frac{8 \pi}{3} \delta(\vec{x})\right), \\
& -3 M\left(\delta_{p a} \frac{x_{b} x_{q}}{r^{5}}-\delta_{q a} \frac{x_{b} x_{p}}{r^{5}}-\delta_{p b} \frac{x_{a} x_{q}}{r^{5}}+\delta_{q b} \frac{x_{a} x_{p}}{r^{5}}\right),
\end{aligned}
$$

and thus $R_{00}=4 \pi G M \delta^{3}(\vec{x}), R_{a b}=4 \pi G M \delta_{a b} \delta^{3}(\vec{x})$. The solution can be translated and boosted to obtain a moving source at an arbitrary location.

\section{Magnetic mass}

We now consider the dual solution, that is, a point magnetic mass sitting at the origin. We have $\Theta^{00}\left(x^{0}, \vec{x}\right)=$ $N \delta^{(3)}(\vec{x})$ as the only non-vanishing component of the magnetic energy-momentum tensor. Furthermore, $T^{\mu \nu}=0$. The solution is linearized Taub-NUT [20], with only magnetic mass, i.e., in polar coordinates,

$$
h_{0 \varphi}=-2 N(1-\cos \theta), \text { other components vanish. }
$$

With this choice of $h_{0 \varphi}$ the string must be taken along the negative $z$-axis in order to cancel the singularity at $\theta=\pi$. The tensor $\Phi_{\lambda}^{\alpha \beta}$ is given by $\Phi^{0 z}{ }_{0}=-16 \pi N \theta(-z) \delta(x) \delta(y)$ (other components vanish).

One then finds the only non-vanishing components (in Cartesian coordinates)

$$
\bar{Y}_{0}^{\prime 0 s}=-2 N \frac{x^{s}}{r^{3}}, \quad \bar{Y}_{c}^{\prime r s}=2 N\left(\delta_{c}^{r} \frac{x^{s}}{r^{3}}-\delta_{c}^{s} \frac{x^{r}}{r^{3}}\right) .
$$

Here, $\bar{Y}_{\gamma}^{\prime \alpha \beta}$ differs from $\bar{Y}_{\gamma}^{\alpha \beta}$ by a gauge transformation (II.14) with $z^{l m}=\epsilon^{l m p} h_{0 p}, z^{0 m}=0$, and hence gives the same curvature. Dealing with $\bar{Y}_{\gamma}^{\alpha \beta}$ rather than $\bar{Y}_{\gamma}^{\alpha \beta}$ simplifies the computations. It follows that the curvature is given by

$$
\begin{aligned}
R_{0 s 0 b} & =0, \quad R_{l m a b}=0 \\
R_{l m 0 b} & =N \epsilon_{l m s}\left(\frac{3 x_{b} x_{s}}{r^{5}}-\frac{\delta_{b s}}{r^{3}}-\frac{4 \pi}{3} \delta_{b s} \delta(\vec{x})\right) \\
R_{0 m a b} & =2 N \epsilon_{a b m}\left(\frac{1}{r^{3}}+\frac{4 \pi}{3} \delta(\vec{x})\right)-3 N\left(\epsilon_{m a k} \frac{x_{b} x_{k}}{r^{5}}-\epsilon_{m b k} \frac{x_{a} x_{k}}{r^{5}}\right),
\end{aligned}
$$

which satisfies the equations of motion, $R_{\alpha \beta}=0$ and $R_{0 i j k}+R_{i j 0 k}+R_{j 0 i k}=4 \pi N \epsilon_{i j k} \delta(\vec{x})=-8 \pi \epsilon_{0 i j \lambda} \bar{\Theta}_{k}^{\lambda}$.

Finally, one easily checks that the linearized Riemann tensor of linearized Taub-NUT is indeed dual to the linearized Riemann tensor of linearized Schwarschild. In that respect, the reason that it was more convenient to work with $\bar{Y}_{\gamma}^{\prime \alpha \beta}$ instead of $\bar{Y}_{\gamma}^{\alpha \beta}$ above is that it is $\bar{Y}_{m a g \gamma}^{\alpha \beta}$ that is dual to $\bar{Y}_{S c h w \gamma}^{\alpha \beta}$. While the curvatures are dual, the original quantities $\bar{Y}_{\gamma}^{\alpha \beta}$ are dual up to a gauge transformation (II.14). 


\section{MAGNETIC SOURCES FOR BOSONIC HIGHER SPINS}

We now indicate how to couple magnetic sources to spins greater than two. The procedure parallels what we have just done for spin 2 but the formulas are somewhat cumbersome because of the extra indices on the fields and the extra trace conditions to be taken into account. We shall therefore only give the general outline of the analysis in the text and relegate the technical details to appendices. The formalism describing higher spin fields in the absence of magnetic sources is recalled in Appendix A.

The spin s curvature $R_{\mu_{1} \nu_{1} \mu_{2} \nu_{2} \cdots \mu_{s} \nu_{s}}$ is the gauge invariant object in terms of which we shall first write the equations of the theory. Its index symmetry is described by the Young tableau

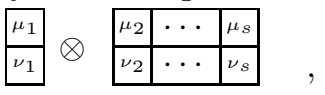

i.e.,

$$
R_{\mu_{1} \nu_{1} \cdots \mu_{i} \nu_{i} \cdots \mu_{s} \nu_{s}}=-R_{\mu_{1} \nu_{1} \cdots \nu_{i} \mu_{i} \cdots \mu_{s} \nu_{s}}, \quad i=1, \cdots, s
$$

and

$$
R_{\mu_{1} \nu_{1} \cdots\left[\mu_{i} \nu_{i} \mu_{i+1}\right] \cdots \mu_{s} \nu_{s}}=0, \quad i=2, \cdots, s-1 .
$$

Its dual, defined through

$$
S_{\mu_{1} \nu_{1} \mu_{2} \nu_{2} \cdots \mu_{s} \nu_{s}}=-\frac{1}{2} \epsilon_{\mu_{1} \nu_{1} \rho \sigma} R_{\mu_{2} \nu_{2} \cdots \mu_{s} \nu_{s}}^{\rho \sigma},
$$

has the same symmetry structure. Note that, just as in the spin 2 case, this does not define an irreducible representation of the linear group. But, also as in the spin 2 case, we shall find that only the irreducible part described by

$$
\begin{array}{|l|l|l|l|}
\hline \mu_{1} & \mu_{2} & \cdots & \mu_{s} \\
\hline \nu_{1} & \nu_{2} & \cdots & \nu_{s} \\
\hline
\end{array}
$$

(i.e., fulfilling also (III.3) for $i=1$ ) corresponds to the independent degrees of freedom (the rest being determined by the sources).

The electric and magnetic energy-momentum tensors will be denoted by $t_{\mu_{1} \mu_{2} \cdots \mu_{s}}$ and $\theta_{\mu_{1} \mu_{2} \cdots \mu_{s}}$. They are conserved, i.e. divergence-free: $\partial_{\mu} t^{\mu \nu_{1} \cdots \nu_{s-1}}=0, \partial_{\mu} \theta^{\mu \nu_{1} \cdots \nu_{s-1}}=0$. Their double traceless parts are written $T_{\mu_{1} \mu_{2} \cdots \mu_{s}}$ and $\Theta_{\mu_{1} \mu_{2} \cdots \mu_{s}}$, and are the tensors that actually couple to the spin s field.

\section{A. Electric and magnetic sources}

The equations in the presence of both electric and magnetic sources are determined again by the requirements: (i) that they reduce to the known equations with electric sources only when the magnetic sources are absent, and (ii) that they be invariant under the duality transformations that rotate the spin s curvature and its dual, as well as the electric and magnetic sources.

Defining $\bar{\Theta}_{\mu_{1} \mu_{2} \cdots \mu_{s}}=\Theta_{\mu_{1} \mu_{2} \cdots \mu_{s}}-\frac{s}{4} \eta_{\left(\mu_{1} \mu_{2}\right.} \Theta_{\left.\mu_{3} \cdots \mu_{s}\right)}^{\prime}$, one finds the following set of equations for the curvature:

$$
\begin{aligned}
R_{\mu_{1} \nu_{1} \mu_{2} \nu_{2} \cdots \mu_{s} \nu_{s}} \eta^{\nu_{1} \nu_{2}} & =\frac{1}{2} \bar{T}_{\mu_{1} \mu_{2}\left[\mu_{3}\left[\cdots\left[\mu_{s}, \nu_{s}\right] \cdots\right] \nu_{3}\right]}, \\
R_{\left[\mu_{1} \nu_{1} \mu_{2}\right] \nu_{2} \cdots \mu_{s} \nu_{s}} & =\frac{1}{6} \epsilon_{\mu_{1} \nu_{1} \mu_{2} \rho} \bar{\Theta}_{\nu_{2}\left[\mu_{3}\left[\cdots\left[\mu_{s}, \nu_{s}\right] \cdots\right] \nu_{3}\right]}^{\rho}, \\
\partial_{[\alpha} R_{\left.\mu_{1} \nu_{1}\right] \mu_{2} \nu_{2} \cdots \mu_{s} \nu_{s}} & =-\frac{1}{3} \epsilon_{\alpha \mu_{1} \nu_{1} \rho} \bar{\Theta}^{\rho}{ }_{\left[\mu_{2}\left[\mu_{3}\left[\cdots\left[\mu_{s}, \nu_{s}\right] \cdots\right] \nu_{3}\right] \nu_{2}\right]} .
\end{aligned}
$$

The first equation is the analog of the Einstein equation (II.5), the second is the analog of the modified cyclic identity (II.1), while the third is the analog of the modified Bianchi identity (II.3). It follows from these equations that the dual curvature obeys similar equations,

$$
\begin{aligned}
S_{\mu_{1} \nu_{1} \mu_{2} \nu_{2} \cdots \mu_{s} \nu_{s}} \eta^{\nu_{1} \nu_{2}} & =\frac{1}{2} \bar{\Theta}_{\mu_{1} \mu_{2}\left[\mu_{3}\left[\cdots\left[\mu_{s}, \nu_{s}\right] \cdots\right] \nu_{3}\right]}, \\
S_{\left[\mu_{1} \nu_{1} \mu_{2}\right] \nu_{2} \cdots \mu_{s} \nu_{s}} & =-\frac{1}{6} \epsilon_{\mu_{1} \nu_{1} \mu_{2} \rho} \bar{T}_{\nu_{2}\left[\mu_{3}\left[\cdots\left[\mu_{s}, \nu_{s}\right] \cdots\right] \nu_{3}\right]}^{\rho}, \\
\partial_{[\alpha} S_{\left.\mu_{1} \nu_{1}\right] \mu_{2} \nu_{2} \cdots \mu_{s} \nu_{s}} & =\frac{1}{3} \epsilon_{\alpha \mu_{1} \nu_{1} \rho} \bar{T}_{\left[\mu_{2}\left[\mu_{3}\left[\cdots\left[\mu_{s}, \nu_{s}\right] \cdots\right] \nu_{3}\right] \nu_{2}\right]}^{\rho},
\end{aligned}
$$

exhibiting manifest duality symmetry. 


\section{B. Decomposition of the curvature tensor}

As in the spin 2 case, the curvature tensor can be expressed in terms of a completely symmetric potential $h_{\mu_{1} \cdots \mu_{s}}$ and of a tensor $\Phi_{\mu_{1} \cdots \mu_{s-1}}^{\rho \sigma}$ fixed by the magnetic energy-momentum tensor, so that the cyclic and Bianchi identities do indeed become identities.

Let $\Phi_{\mu_{1} \cdots \mu_{s-1}}^{\rho \sigma}$ be such that

$$
\partial_{\rho} \Phi_{\mu_{1} \cdots \mu_{s-1}}^{\rho \sigma}=\theta_{\mu_{1} \cdots \mu_{s-1}}^{\sigma}
$$

and let $\hat{\Phi}_{\mu_{1} \cdots \mu_{s-1}}^{\rho \sigma}$ be the part of $\Phi_{\mu_{1} \cdots \mu_{s-1}}^{\rho \sigma}$ that is traceless in the indices $\mu_{1} \cdots \mu_{s-1}$. For computations, it is useful to note that $\partial_{\rho} \hat{\Phi}^{\rho \sigma}{ }_{\mu_{1} \cdots \mu_{s-1}}=\Theta^{\sigma}{ }_{\mu_{1} \cdots \mu_{s-1}}-\frac{(s-2)}{4} \eta_{\left(\mu_{1} \mu_{2}\right.} \Theta_{\left.\mu_{3} \cdots \mu_{s-1}\right)}^{\prime \sigma}$. The expression of the tensor $\Phi^{\rho \sigma}{ }_{\mu_{1} \cdots \mu_{s-1}}$ in terms of the Dirac string is given below. The appropriate expression of the curvature tensor in terms of the spin $\mathrm{s}$ field and the Dirac string contribution is:

$$
R_{\mu_{1} \nu_{1} \mu_{2} \nu_{2} \cdots \mu_{s} \nu_{s}}=-\frac{1}{2} \epsilon_{\mu_{1} \nu_{1} \rho \sigma} \bar{Y}_{\left[\mu_{2}\left[\mu_{3}\left[\cdots\left[\mu_{s}, \nu_{s}\right] \cdots\right] \nu_{3}\right] \nu_{2}\right]}^{\rho \sigma}
$$

where

$$
\begin{aligned}
\bar{Y}_{\mu_{1} \cdots \mu_{s-1}}^{\rho \sigma} & =Y_{\mu_{1} \cdots \mu_{s-1}}^{\rho \sigma}+\frac{2(s-1)}{s} \delta_{\left(\mu_{1}\right.}^{[\rho} Y_{\left.\mu_{2} \cdots \mu_{s-1}\right) \theta}^{\sigma] \theta}, \\
Y_{\mu_{1} \cdots \mu_{s-1}}^{\rho \sigma} & =\partial_{\theta} X_{\mu_{1} \cdots \mu_{s-1}}^{\rho \sigma \theta}+\hat{\Phi}_{\mu_{1} \cdots \mu_{s-1}}^{\rho \sigma}, \\
X_{\mu_{1} \cdots \mu_{s-1}}^{\rho \sigma \theta} & =\epsilon^{\rho \sigma \theta \lambda} h_{\lambda \mu_{1} \cdots \mu_{s-1}}-\frac{3(s-1)(s-2)}{2 s} \eta_{\alpha\left(\mu_{1}\right.} \delta_{\mu_{2}}^{[\rho} \epsilon^{\sigma \theta] \alpha \beta} h_{\left.\mu_{3} \cdots \mu_{s-1}\right) \beta}^{\prime} .
\end{aligned}
$$

The split of $Y_{\mu_{1} \cdots \mu_{s-1}}^{\rho \sigma}$ into an $X$-part and a $\Phi$-part defines a split of the Riemann tensor analogous to the split (II.7) introduced for spin 2. The Dirac string contribution ( $\Phi$-term) removes the magnetic terms violating the standard cyclic and Bianchi identities, leaving one with a tensor $r_{\mu_{1} \nu_{1} \mu_{2} \nu_{2} \cdots \mu_{s} \nu_{s}}$ that fulfills

$$
r_{\left[\mu_{1} \nu_{1} \mu_{2}\right] \nu_{2} \cdots \mu_{s} \nu_{s}}=0, \quad \partial_{[\alpha} r_{\left.\mu_{1} \nu_{1}\right] \mu_{2} \nu_{2} \cdots \mu_{s} \nu_{s}}=0
$$

and thus derives from a symmetric potential (the spin s field $h_{\mu_{1} \cdots \mu_{s}}$ ) as

$$
r_{\mu_{1} \nu_{1} \mu_{2} \nu_{2} \cdots \mu_{s} \nu_{s}}=-2 h_{\left[\mu_{1}\left[\mu_{2} \cdots\left[\mu_{s}, \nu_{s}\right] \cdots \nu_{2}\right] \nu_{1}\right]}
$$

(see Appendix A). The $X$-term in the curvature is a rewriting of (III.16) that is convenient for the subsequent analysis. The potential $h_{\mu_{1} \cdots \mu_{s}}$ is determined from the curvature up to a gauge transformation with unconstrained trace. The fact that only $\hat{\Phi}_{\mu_{1} \cdots \mu_{s-1}}^{\rho \sigma}$ appears in the curvature and not $\Phi^{\rho \sigma}{ }_{\mu_{1} \cdots \mu_{s-1}}$ is a hint that only the double traceless part $\Theta_{\mu_{1} \cdots \mu_{s}}$ of the magnetic energy-momentum tensor plays a physical role.

\section{Equations of motion for the spin s field}

In terms of the potential, the remaining equation (III.5) is of order s. In the sourceless case, one replaces it by a second order equation written first by Fronsdal [11]. This can be done also in the presence of both electric and magnetic sources by following the procedure described in $[14,15]$. The crucial observation is that the curvature is related as in (A.5), namely,

$$
R_{\mu_{1} \nu_{1} \mu_{2} \nu_{2} \cdots \mu_{s} \nu_{s}} \eta^{\nu_{1} \nu_{2}}=-\frac{1}{2} F_{\mu_{1} \mu_{2}\left[\mu_{3}\left[\cdots\left[\mu_{s}, \nu_{s}\right] \cdots\right] \nu_{3}\right]}
$$

to the generalized Fronsdal tensor given by

$$
F_{\gamma_{1} \cdots \gamma_{s}}=-\frac{1}{2} \epsilon_{\gamma_{1} \mu \nu \lambda}\left(\partial^{\lambda} \bar{Y}_{\gamma_{2} \cdots \gamma_{s}}^{\mu \nu}-(s-1) \partial_{\left(\gamma_{2}\right.} \bar{Y}_{\left.\gamma_{3} \cdots \gamma_{s}\right)}^{\mu \nu \lambda}\right)
$$

so that (III.5) is equivalent to $F_{\mu_{1} \mu_{2}\left[\mu_{3}\left[\cdots\left[\mu_{s}, \nu_{s}\right] \cdots\right] \nu_{3}\right]}+\bar{T}_{\mu_{1} \mu_{2}\left[\mu_{3}\left[\cdots\left[\mu_{s}, \nu_{s}\right] \cdots\right] \nu_{3}\right]}=0$. This implies $F_{\mu_{1} \mu_{2} \mu_{3} \cdots \mu_{s}}+\bar{T}_{\mu_{1} \mu_{2} \mu_{3} \cdots \mu_{s}}=$ $\partial_{\left(\mu_{1} \mu_{2} \mu_{3}\right.} \Lambda_{\left.\mu_{4} \cdots \mu_{s}\right)}$ for some $\Lambda_{\mu_{4} \cdots \mu_{s}}$ [22]. By making a gauge transformation on the spin s field, one can set the right-hand side of this relation equal to zero (see Appendix A), obtaining the field equation

$$
F_{\mu_{1} \mu_{2} \mu_{3} \cdots \mu_{s}}+\bar{T}_{\mu_{1} \mu_{2} \mu_{3} \cdots \mu_{s}}=0
$$


which fixes the trace of the gauge parameter. When $\mathrm{s}=3$ this is the end of the story.

For $\mathrm{s} \geq 4$ additional restrictions are necessary, namely, we shall demand that the gauge transformation that brings the field equation to the form (III.19) eliminates at the same time the double trace of the field $h_{\mu_{1} \cdots \mu_{s}}$ (see [14] for a discussion).

In terms of the generalized Einstein tensor defined as in (A.7), i.e.,

$$
G_{\mu_{1} \mu_{2} \cdots \mu_{s}}=F_{\mu_{1} \mu_{2} \cdots \mu_{s}}-\frac{s(s-1)}{4} \eta_{\left(\mu_{1} \mu_{2}\right.} F_{\left.\mu_{3} \cdots \mu_{s}\right) \rho}{ }^{\rho},
$$

the equations become

$$
G_{\mu_{1} \mu_{2} \mu_{3} \cdots \mu_{s}}+T_{\mu_{1} \mu_{2} \mu_{3} \cdots \mu_{s}}=0 .
$$

We shall thus adopt (III.21), with the Einstein tensor, Fronsdal tensor and $Y$-tensor defined as in (III.20), (III.18) and (III.13), respectively, as the equations of motion for a double traceless spin s field $h_{\mu_{1} \cdots \mu_{s}}$. These equations imply (III.5) through (III.10) and define the theory in the presence of both electric and magnetic sources. It is these equations that we shall derive from a variational principle.

\section{Point particles sources - Dirac string}

For point sources, the tensors that couple to the spin s field read

$$
t^{\mu \nu_{1} \cdots \nu_{s-1}}=M u^{\nu_{1}} \cdots u^{\nu_{s-1}} \int d \lambda \delta^{(4)}(x-z(\lambda)) \dot{z}^{\mu}=M \frac{u^{\mu} u^{\nu_{1}} \cdots u^{\nu_{s-1}}}{u^{0}} \delta^{(3)}\left(\vec{x}-\vec{z}\left(x^{0}\right)\right)
$$

and

$$
\theta^{\mu \nu_{1} \cdots \nu_{s-1}}=N v^{\nu_{1}} \cdots v^{\nu_{s-1}} \int d \lambda \delta^{(4)}(x-\bar{z}(\lambda)) \dot{\bar{z}}^{\mu}=N \frac{v^{\mu} v^{\nu_{1}} \cdots v^{\nu_{s-1}}}{v^{0}} \delta^{(3)}\left(\vec{x}-\vec{z}\left(x^{0}\right)\right) .
$$

One can check that they are indeed conserved.

A tensor $\Phi_{\gamma_{1} \cdots \gamma_{s-1}}^{\alpha \beta}$ that satisfies (III.11) can again be constructed by attaching a Dirac string $y^{\mu}(\lambda, \sigma)$ to the magnetic source, $y^{\mu}(\lambda, 0)=\bar{z}^{\mu}(\lambda)$. One has

$$
\Phi_{\gamma_{1} \cdots \gamma_{s-1}}^{\alpha \beta}=N v_{\gamma_{1}} \cdots v_{\gamma_{s-1}} \int d \lambda d \sigma\left(y^{\prime \alpha} \dot{y}^{\beta}-\dot{y}^{\alpha} y^{\prime \beta}\right) \delta^{(4)}(x-y(\lambda, \sigma)) .
$$

One can compute explicitly the conserved charges associated with asymptotic symmetries for electric point sources (see Appendix A). Using the equations of motion, they read

$$
P^{\mu_{1} \cdots \mu_{s-1}}=M f^{\mu_{1} \cdots \mu_{s-1}}(u),
$$

where $f^{\mu_{1} \cdots \mu_{s-1}}(u)$ is the traceless part of $u^{\mu_{1}} \cdots u^{\mu_{s-1}}$. One then finds

$$
f^{\mu_{1} \cdots \mu_{s-1}}(u)=\sum_{l} \alpha_{l} \eta^{\left(\mu_{1} \mu_{2}\right.} \cdots \eta^{\mu_{2 l-1} \mu_{2 l}} u^{\mu_{2 l+1}} \cdots u^{\left.\mu_{s-1}\right)}|u|^{2 l},
$$

where the sum goes over $l=0,1, \cdots$ such that $2 l \leq s-1, \alpha_{0}=1$ and $\alpha_{l+1}=-\frac{(s-1-2 l)(s-2-2 l)}{4(l+1)(s-1-l)} \alpha_{l}$.

The dual magnetic charges

$$
Q^{\mu_{1} \cdots \mu_{s-1}}=N f^{\mu_{1} \cdots \mu_{s-1}}(v)
$$

are also conserved.

\section{E. Variational Principle}

The second-order equations of motion $G_{\gamma_{1} \cdots \gamma_{s}}+T_{\gamma_{1} \cdots \gamma_{s}}=0$ equivalent to (III.5), are the Euler-Lagrange derivatives with respect to $h^{\gamma_{1} \cdots \gamma_{s}}$ of the action

$$
S\left[h_{\mu_{1} \cdots \mu_{s}}(x), y^{\mu}(\lambda, \sigma)\right]=\int d^{4} x\left(\mathcal{L}+h_{\mu_{1} \cdots \mu_{s}} t^{\mu_{1} \cdots \mu_{s}}\right),
$$


where

$$
\begin{aligned}
\mathcal{L}=-\frac{(s-1)}{2} Y_{\mu \nu \alpha_{1} \cdots \alpha_{s-1}}\left[-Y^{\mu \alpha_{1} \nu \alpha_{2} \cdots \alpha_{s-1}}+\frac{(s-2)}{2(s-1)} Y^{\mu \nu \alpha_{1} \cdots \alpha_{s-1}}\right. & \\
& \left.+\frac{(s-3)}{s} \eta^{\mu \alpha_{1}} Y^{\nu \rho \alpha_{2} \cdots \alpha_{s-1}}{ }_{\rho}-\frac{(s-2)}{s} \eta^{\mu \alpha_{1}} Y^{\alpha_{2} \rho \nu \alpha_{3} \cdots \alpha_{s-1}}{ }_{\rho}\right] .
\end{aligned}
$$

Indeed, one can check that $\frac{\delta \mathcal{L}}{\delta \gamma^{\gamma_{1} \cdots \gamma_{s}}}=G_{\gamma_{1} \cdots \gamma_{s}}$. As in the spin 2 case, the trajectories of the electric and magnetic sources are kept fixed, i.e., the sources are not dynamical. The magnetic coupling in the action was obtained by introducing the familiar minimal electric coupling in the "parent action" of [16], which contains two potentials, and determining what it becomes in the dual formulation.

The action (III.22) can be verified to be invariant under the gauge symmetries (A.1) of the spin s field as well as under displacements of the Dirac string (accompanied by an appropriate redefinition of $h_{\mu_{1} \cdots \mu_{s}}$ ). This is done in Appendix B.

\section{F. Quantization condition}

As for spin 2, the unobservability of the Dirac string in the quantum theory leads to a quantization condition. The computation proceeds exactly as in the spin 2 case and is reproduced in Appendix C. One finds explicitly the quantization condition

$$
\frac{M N}{2 \pi \hbar} f_{\gamma_{1} \cdots \gamma_{s-1}}(v) f^{\gamma_{1} \cdots \gamma_{s-1}}(u)=n, \quad n \in \mathbb{Z} .
$$

In terms of the conserved charges $P^{\gamma_{1} \cdots \gamma_{s-1}}$ associated with the asymptotic symmetries and their dual charges $Q^{\gamma_{1} \cdots \gamma_{s-1}}$, the quantization condition can be rewritten as

$$
\frac{1}{2 \pi \hbar} Q_{\gamma_{1} \cdots \gamma_{s-1}}(v) P^{\gamma_{1} \cdots \gamma_{s-1}}(u) \in \mathbb{Z}
$$

\section{BEYOND THE LINEAR THEORY FOR SPIN TWO}

We have seen that magnetic sources can be introduced for linearized gravity and linearized higher-spin theories, and that an appropriate generalization of the Dirac quantization condition on the sources must hold. However in the linear theory the treatment is already incomplete since the sources must be external. In the full non linear theory even the introduction of external sources is not possible. For spin 2 the difficulty stems from the fact that the source must be covariantly conserved and for spins $\geq 2$ the formulation of the non linear theory is still incomplete.

Nevertheless, we shall address the issue of duality in the spin 2 case, for which the non linear theory in the absence of sources is the vacuum Einstein theory of gravitation. This is the "electric" formulation. Electric sources are coupled through their standard energy-momentum momentum tensor. We do not know whether magnetic sources should appear as independent fundamental degrees of freedom (the complete action with these degrees of freedom included is unknown and would presumably be non local, as the results of [23] suggest) or whether they should appear as solitons somewhat like in Yang-Mills theory [5].

Whatever the answer, there are indications that duality invariance and quantization conditions are valid beyond the flat space, linear regime studied above. One indication is given by dimensional reduction of the full Einstein theory, which reveals the existence of "hidden symmetries" that include duality [24]. Another indication that non linear gravity enjoys duality invariance is given by the existence of the Taub-NUT solution [20], which is an exact solution of the vacuum Einstein theory describing a gravitational dyon. The quantization condition on the energy of a particle moving in the Taub-NUT geometry is a well known result which has been discussed by many authors [25] and which can be viewed as a consequence of the existence of closed timelike lines [26].

Because of the importance of the Taub-NUT metric, we shall devote the rest of the paper to a further investigation of its properties. First, we shall rederive the quantization condition along new lines, from the quantization of the angular momentum stored in the gravitational field. Next, we compute the Poincare charges for the Taub-NUT metric, which is asymptotically flat at spatial infinity in the sense of [27]. 


\section{A. The gravitating magnetic pole}

The Taub-NUT metric is given by :

$$
d s^{2}=-V(r)[d t+2 N(k-\cos \theta) d \phi]^{2}+V(r)^{-1} d r^{2}+\left(r^{2}+N^{2}\right)\left(d \theta^{2}+\sin ^{2} \theta d \phi^{2}\right),
$$

with

$$
V(r)=1-\frac{2\left(N^{2}+M r\right)}{\left(r^{2}+N^{2}\right)}=\frac{r^{2}-2 M r-N^{2}}{r^{2}+N^{2}}
$$

where $N$ and $M$ are the magnetic and electric masses as follows from the asymptotic analysis of the metric and our discussion of the linear theory. A pure magnetic mass has $M=0$. The number $k$ can be changed according to

$$
k \rightarrow k^{\prime}=k-\alpha
$$

by performing a $t$ coordinate transformation

$$
t \rightarrow t^{\prime}=t+2 N \alpha \phi
$$

The metric (IV.1) is singular on the $z$-axis. This singularity is most easily seen by calculating $|\nabla t|^{2}$ and can be interpreted as a singularity of the metric or of the $t$ coordinate. It is known in the literature as the Dirac-Misner string singularity, due to its analogy with the Maxwell case. Its location depends on the value of $k$ : for $k=1$ the singularity is at $\theta=\pi$ and for $k=-1$ it is at $\theta=0$. For all other values of $k$ both string singularities exist. The choice $k=0$ makes the North and South poles play a symmetrical role. In his paper [26], Misner showed that the singularity is a coordinate singularity and that the metric describes a non-singular manifold provided that the time coordinate $t$ is taken to be periodic with period $8 \pi N$.

\section{B. Spatial rotations and quantization condition}

Even though the metric contains a $d t d \phi$ term, it is spherically symmetric. However, the rotation group acts on spacetime in an unconventional way [26] and the rotation Killing vectors differ from those of flat space by extra terms. One can understand the origin of these extra terms by comparing the Taub-NUT solution with the standard electromagnetic magnetic monopole solution and recalling that for stationary metrics, the mixed time-space metric components are naturally interpreted as the component of an electromagnetic vector potential ("gravitomagnetism"): $g_{0 i} \sim A_{i}$. In this spirit, the above metric component $g_{0 i}$ would correspond to a monopole potential

$$
A_{\phi}=-2 N(k-\cos \theta)
$$

with magnetic charge $N$, as has been observed by many authors.

To understand the form of the Killing vectors, we start with the generator of rotations around the $z$-axis. It is recalled in Subsection D 1 that in the electromagnetic case, rotations of the electromagnetic potential of a magnetic pole must be accompanied by gauge transformations. When the $g_{0 i}$ metric components are interpreted as the components of a vector potential, the electromagnetic gauge transformations lift to diffeomorphisms along the time direction. We therefore expect the gauge parameter which accompanies a rotation to lift to a component along $\partial / \partial t$.

This expectation turns out to be correct. The metric (IV.1) has four Killing vectors, given in [26] for $k=1$, which we display as

$$
\begin{aligned}
\xi_{t} & =\frac{\partial}{\partial t} \\
\xi_{x} & =-\sin \phi \frac{\partial}{\partial \theta}-\cos \phi \cot \theta \frac{\partial}{\partial \phi}+\left(2 N k \cos \phi \cot \theta-2 N \frac{\cos \phi}{\sin \theta}\right) \frac{\partial}{\partial t} \\
\xi_{y} & =\cos \phi \frac{\partial}{\partial \theta}-\sin \phi \cot \theta \frac{\partial}{\partial \phi}+\left(2 N k \sin \phi \cot \theta-2 N \frac{\sin \phi}{\sin \theta}\right) \frac{\partial}{\partial t} \\
\xi_{z} & =\frac{\partial}{\partial \phi}-2 N k \frac{\partial}{\partial t}
\end{aligned}
$$

These satisfy the commutation relations

$$
\left[\xi_{a}, \xi_{b}\right]=-\epsilon_{a b c} \xi_{c}, \quad\left[\xi_{a}, \xi_{t}\right]=0,
$$


where $a, b, c=x, y, z$ and $\epsilon_{x y z}=1$, which constitute the standard $\operatorname{su}(2) \times u(1)$ Lie algebra. For $N=0$, which is the Schwarzschild case, the three Killing vectors $\xi_{a}$ generate spacelike two-spheres. In the case of Taub-NUT, although the algebra is the same, the action of the group on the manifold is, as pointed out by Misner, different. In fact, the $\xi_{a}$ which satisfy the $\mathrm{su}(2)$ algebra now generate the $r=$ constant three-spheres. These three-spheres have a Lorentzian metric.

The three rotation Killing vectors $\xi_{a}$ are invariant under reflections with respect to the origin $(t \rightarrow-t, \theta \rightarrow \pi-\theta$, $\phi \rightarrow \phi+\pi, N \rightarrow-N$ and $k \rightarrow-k)$. This should be the case because being the generators of rotations they are pseudo-vectors.

One also observes that $\xi_{z}$ has a component along $\partial / \partial t$ proportional to the magnetic mass. We find this fact very appealing, as it leads to a different derivation of the quantization condition as we now show.

Consider an electric mass following a geodesic in the Taub-NUT spacetime, with four-momentum $p_{\mu}=m u_{\mu}$. There exists a conserved charge associated with every Killing vector field, and in particular a charge $J_{Z}$ associated with $\xi_{z}$ :

$$
J_{Z}=m \xi_{z}^{\mu} u_{\mu}=p_{\phi}-2 N m u_{0} k
$$

We see that just as in the electromagnetic case, the angular momentum about the z-axis has an extra piece besides the standard $p_{\phi}$. This extra piece comes from the angular momentum in the field, which varies as the particle moves and which must be taken into account in the conservation law. In fact, the extra term coincides with the angular momentum in the field when $k=1$ and the particle is on the positive $z$-axis (see Appendix D). Thus the angular momentum in the field is equal to $2 N m u_{0}$. Requiring this angular momentum to be quantized in multiples of $\hbar / 2$ yields the quantization condition

$$
4 N m u_{0} \in \hbar \mathbb{Z}
$$

Note that when the particle is on the negative $z$-axis, the angular momentum in the field coincides with the extra term in $J_{Z}$ when $k=-1$ and therefore changes sign.

Formula (IV.9) agrees with the condition that comes from periodicity in time of the wave function. Suppose indeed that the electric mass is described by a wavefunction $\psi$. The time dependence of $\psi$ is given by

$$
\psi \propto e^{-\frac{i E t}{\hbar}}
$$

where $E=m u_{0}$ is the energy of the particle. Recalling that time is periodic with period $\Delta t=8 \pi N$, and requiring single valuedness of the wave function, we obtain $E \Delta t=2 n \pi \hbar$ which implies, yet again, that

$$
4 N m u_{0} \in \hbar \mathbb{Z}
$$

Single-valuedness is required because the closed timelike curves are contractible.

At this point we may resort to the concept of duality invariance to argue that the result should also hold if we were considering a test magnetic mass in the gravitational field of an electric mass, or in fact both. Thus we are led to the conclusion that the quantization conditions derived in the linear theory

$$
\frac{4 G\left(P_{\gamma} \bar{Q}^{\gamma}-\bar{P}_{\gamma} Q^{\gamma}\right)}{\hbar} \equiv \frac{4 G \epsilon_{a b} Q_{\gamma}^{a} \bar{Q}^{b \gamma}}{\hbar} \in Z
$$

also holds in the full non linear theory. The scalar product which appears here is the Minkowskian scalar product of the asymptotic charges $Q_{\gamma}^{a}$ and $\bar{Q}^{b \gamma}$. The antisymmetry of the quantization condition for the exchange of the dyons is confirmed by the change of sign of the angular momentum stored in the field which was observed above.

\section{Taub-NUT is asymptotically flat at spatial infinity}

The introduction of the Minkowskian scalar product is legitimate because the Taub-NUT metric (IV.1) is asymptotically flat at spatial infinity in the sense of [27]. In polar coordinates, the conditions of [27], including their parity conditions, read $h_{r r}=O\left(r^{-1}\right), h_{r \theta}=O(1), h_{r \phi}=O(1), h_{\theta \theta}=O(r), h_{\theta \phi}=O(r), h_{\phi \phi}=O(r)$ and the leading terms (of the order just written) in $h_{r r}, h_{r \phi}, h_{\theta \theta}, h_{\phi \phi}$ should be even under the inversion $\theta \rightarrow \pi-\theta$ and $\phi \rightarrow \phi+\pi$, while the leading terms in $h_{r \theta}, h_{\theta \phi}$ should be odd. Here, $h_{i j}$ is the deviation from the flat metric also written in polar coordinates. Similarly, the conjugate momenta should fulfill $\pi^{r r}=O(1), \pi^{r \theta}=O\left(r^{-1}\right), \pi^{r \phi}=O\left(r^{-1}\right), \pi^{\theta \theta}=O\left(r^{-2}\right)$, $\pi^{\theta \phi}=O\left(r^{-2}\right), \pi^{\phi \phi}=O\left(r^{-2}\right)$, with the parity conditions that the leading terms in $\pi^{r r}, \pi^{r \phi}, \pi^{\theta \theta}$ and $\pi^{\phi \phi}$ are odd, while those in $\pi^{r \theta}$ and $\pi^{\theta \phi}$ are even. 
It is easy to see that all these conditions are fulfilled in the coordinate system with $k=0$, where the Taub-NUT metric reads

$$
d s^{2}=-V(r)[d t-2 N \cos \theta d \phi]^{2}+V(r)^{-1} d r^{2}+\left(r^{2}+N^{2}\right)\left(d \theta^{2}+\sin ^{2} \theta d \phi^{2}\right) .
$$

The perturbation $h_{i j}$ is not smooth as a tensor on the sphere because of the string singularities at $\theta=0, \pi$ where $h_{\phi \phi}$ does not vanish, but its behavior as a function of $r$ is nevertherless compatible with the required asymptotic fall-off. This guarantees finiteness of all the surface integrals for the Poincaré charges because the parity conditions are in particular fulfilled. Coordinate systems with $k \neq 0$ fail to fulfill the parity conditions and for this reason, are not amenable to the treatment of [27].

This notion of asymptotic flatness appears to be all that is needed for the action principle and the identification of conserved charges. It does not, and need not, eliminate, of course, the geometrical difficulties pointed out in [26] in regard, for example, to the probable impossibility of writing the metric at large distances as a smooth perturbation of Minkowski space.

Note that it is not necessary to fix the magnetic mass $N$ at infinity. Competing histories in the variational principle may have different $N$ 's. This treats $M$ and $N$ on an equal footing, preserving duality [34]. It is at first sight disturbing that the Killing vectors (IV.6) depend on $N$ because it would seem that the asymptotic symmetry group depends on the magnetic mass which as we just noted can be varied. There is however no difficulty because the surface integrals associated with the $N$ dependent terms in the killing vectors vanish on account of the parity conditions. Indeed as it was observed above since $N$ is a pseudo-scalar the angular dependence of the factor that multiplies it in the rotation Killing vectors is also odd under inversions as required by the parity conditions.

If one computes the Poincaré charges for the metric (IV.13) using the formulas of [27], one finds

$$
P^{0}=M, \quad P^{i}=0, \quad J_{i j}=0, \quad J_{0 i}=0 .
$$

The parameter $N$ does not contribute to the "electric" Poincaré charges. Observe in particular that the angular momentum is zero for the Taub-NUT solution, indicating that the solution may be thought of as describing a dyon with the two charges on top of one another. If the magnetic mass and electric mass are separated, there is a net angular momentum and the metric belongs to the Kerr-Newman-Taub-NUT [28] family, which describes metrics with angular momentum and electromagnetic charges. These metrics fulfill also the boundary conditions of [27]. Other asymptotic analysis of the Taub-NUT solution (at null infinity) may be found in [29].

\section{CONCLUSIONS}

In this paper we have extended to all integer spins the action for magnetic poles introduced by Dirac. The construction has two limitations: (i) it deals only with the linearized field theory and (ii) the sources must be external. The presumption is however strong that, at least in the spin 2 case, that is, the theory of gravitation, monopoles exist and the corresponding Dirac quantization condition holds. Arguments in this direction are advanced based on properties of Taub-NUT space. The analogy with electromagnetism is emphasized throughout.

\section{Acknowledgments}

MH is grateful to KITP for hospitality while the article was being prepared. This work was funded by an institutional grant to CECS of the Millennium Science Initiative, Chile, and Fundación Andes, and also benefits from the generous support to CECS by Empresas CMPC. The work of SC and MH is partially supported by IISN - Belgium (convention 4.4505.86), by the "Interuniversity Attraction Monopoles Programme - Belgian Science Policy " and by the European Commission programme MRTN-CT-2004-005104, in which they are associated to V.U. Brussel. RP gratefully acknowledges support from FONDECYT grant 3050086. This research was also supported in part by the National Science Foundation under Grant No. PHY99-07949. SC is Aspirant du FNRS (Belgium). 


\section{APPENDIX A: HIGHER SPIN GAUGE FIELDS IN THE ABSENCE OF MAGNETIC SOURCES}

We recall in this appendix the free theory of bosonic higher spin fields.

\section{Spin s field and gauge invariance}

In the absence of sources, a massless bosonic spin $s$ field is described by a gauge potential which is a totally symmetric tensor $h_{\mu_{1} \mu_{2} \cdots \mu_{s}}$ subject to the "double-tracelessness condition" [11], $h_{\mu_{1} \mu_{2} \cdots \mu_{s}}=$ $h_{\left(\mu_{1} \mu_{2} \cdots \mu_{s}\right)}, \quad h_{\mu_{1} \mu_{2} \mu_{3} \mu_{4} \cdots \mu_{s}} \eta^{\mu_{1} \mu_{2}} \eta^{\mu_{3} \mu_{4}}=0$. The gauge transformation reads

$$
h_{\mu_{1} \mu_{2} \cdots \mu_{s}} \rightarrow h_{\mu_{1} \mu_{2} \cdots \mu_{s}}+\partial_{\left(\mu_{1}\right.} \xi_{\left.\mu_{2} \cdots \mu_{s}\right)},
$$

where the gauge parameter $\xi_{\mu_{2} \cdots \mu_{s}}$ is traceless, $\xi_{\mu_{2} \mu_{3} \cdots \mu_{s}} \eta^{\mu_{2} \mu_{3}}=0$. The trace condition on the gauge parameter appears for spins $\geq 3$, while the double tracelessness condition on the field appears for spins $\geq 4$.

From the field $h_{\mu_{1} \mu_{2} \cdots \mu_{s}}$, one can construct a curvature $R_{\mu_{1} \nu_{1} \mu_{2} \nu_{2} \cdots \mu_{s} \nu_{s}}$ that contains $s$ derivatives of the field and that is gauge invariant under the transformations (A.1) even if the gauge parameter is not traceless,

$$
R_{\mu_{1} \nu_{1} \mu_{2} \nu_{2} \cdots \mu_{s} \nu_{s}}=-2 h_{\left[\mu_{1}\left[\mu_{2} \cdots\left[\mu_{s}, \nu_{s}\right] \cdots \nu_{2}\right] \nu_{1}\right]},
$$

where one antisymmetrizes over $\mu_{k}$ and $\nu_{k}$ for each $k$. This is the analog of the Riemann tensor of the spin 2 case. The curvature $R_{\mu_{1} \nu_{1} \mu_{2} \nu_{2} \cdots \mu_{s} \nu_{s}}$ has the symmetry characterized by the Young tableau

$$
\begin{array}{|l|l|l|l|}
\hline \mu_{1} & \mu_{2} & \cdots & \mu_{s} \\
\hline \nu_{1} & \nu_{2} & \cdots & \nu_{s} \\
\hline
\end{array}
$$

and fulfills the Bianchi identity

$$
\partial_{[\alpha} R_{\left.\mu_{1} \nu_{1}\right] \mu_{2} \nu_{2} \cdots \mu_{s} \nu_{s}}=0 \text {. }
$$

Conversely, given a tensor $R_{\mu_{1} \nu_{1} \mu_{2} \nu_{2} \cdots \mu_{s} \nu_{s}}$ with the Young tableau symmetry (A.3) and fulfilling the Bianchi identity (A.4), there exists a "potential" $h_{\mu_{1} \mu_{2} \cdots \mu_{s}}$ such that (A.2) holds. This potential is determined up to a gauge transformation (A.1) where the gauge parameter $\xi_{\mu_{2} \cdots \mu_{s}}$ is unconstrained [22].

\section{Equations of motion}

The trace conditions on the gauge parameter for spins $\geq 3$ are necessary in order to construct second-order invariants - and thus, in particular, gauge invariant second-order equations of motion. One can show that the Fronsdal tensor

$$
F_{\mu_{1} \mu_{2} \cdots \mu_{s}}=\partial^{\rho} \partial_{\rho} h_{\mu_{1} \mu_{2} \cdots \mu_{s}}-s \partial_{\left(\mu_{1}\right.} \partial^{\rho} h_{\left.\mu_{2} \cdots \mu_{s}\right) \rho}+\frac{s(s-1)}{2} \partial_{\left(\mu_{1} \mu_{2}\right.} h_{\left.\mu_{3} \cdots \mu_{s}\right) \rho}{ }^{\rho},
$$

which contains only second derivatives of the potential, transforms under a gauge transformation (A.1) into the trace of the gauge parameter

$$
F_{\mu_{1} \mu_{2} \cdots \mu_{s}} \rightarrow F_{\mu_{1} \mu_{2} \cdots \mu_{s}}+\frac{(s-1)(s-2)}{2} \partial_{\left(\mu_{1} \mu_{2} \mu_{3}\right.} \xi_{\left.\mu_{4} \cdots \mu_{s}\right) \rho}{ }^{\rho},
$$

and is thus gauge invariant when the gauge parameter is requested to be traceless. The Fronsdal tensor is related to the curvature by the relation

$$
R_{\mu_{1} \nu_{1} \mu_{2} \nu_{2} \cdots \mu_{s} \nu_{s}} \eta^{\nu_{1} \nu_{2}}=-\frac{1}{2} F_{\mu_{1} \mu_{2}\left[\mu_{3}\left[\cdots\left[\mu_{s}, \nu_{s}\right] \cdots\right] \nu_{3}\right]} .
$$

In the absence of sources the equations of motion that follow from the variational principle written in the main text (III.22) are

$$
G_{\mu_{1} \mu_{2} \cdots \mu_{s}}=0
$$

where the "Einstein" tensor is defined as

$$
G_{\mu_{1} \mu_{2} \cdots \mu_{s}}=F_{\mu_{1} \mu_{2} \cdots \mu_{s}}-\frac{s(s-1)}{4} \eta_{\left(\mu_{1} \mu_{2}\right.} F_{\left.\mu_{3} \cdots \mu_{s}\right) \rho}^{\rho} .
$$


They obviously imply

$$
R_{\mu_{1} \nu_{1} \mu_{2} \nu_{2} \cdots \mu_{s} \nu_{s}} \eta^{\nu_{1} \nu_{2}}=0
$$

and the inverse implication is true as well [15]. Indeed, Eq. (A.8) implies that the Fronsdal tensor has the form $F_{\mu_{1} \mu_{2} \cdots \mu_{s}}=\partial_{\left(\mu_{1} \mu_{2} \mu_{3}\right.} \Sigma_{\left.\mu_{4} \cdots \mu_{s}\right)}$, which can be made to vanish by a gauge transformation with an unconstrained gauge parameter (see [14] for a discussion of the subtleties associated with the double tracelessness of the spin s field $h_{\mu_{1} \ldots \mu_{s}}$ ). The interest of the equations (A.8) derived from the Einstein equations is that they contain the same number of derivatives as the curvature. Thus, they are useful to exhibit duality, which rotates the equations of motion and the cyclic identities on the curvature.

\section{Dual curvature}

The dual of the curvature tensor is defined by

$$
S_{\mu_{1} \nu_{1} \mu_{2} \nu_{2} \cdots \mu_{s} \nu_{s}}=-\frac{1}{2} \epsilon_{\mu_{1} \nu_{1} \rho \sigma} R_{\mu_{2} \nu_{2} \cdots \mu_{s} \nu_{s}}^{\rho \sigma},
$$

and, as a consequence of the equations of motion (A.8), of the symmetry of the curvature and of the Bianchi identity (A.4), it has the same symmetry as the curvature and fulfills the equations $S_{\mu_{1} \nu_{1} \mu_{2} \nu_{2} \cdots \mu_{s} \nu_{s}} \eta^{\nu_{1} \nu_{2}}=0$, $\partial_{[\alpha} S_{\left.\mu_{1} \nu_{1}\right] \mu_{2} \nu_{2} \cdots \mu_{s} \nu_{s}}=0$.

\section{Conserved charges}

Non vanishing conserved charges can be associated with the gauge transformations (A.1) that tend to Killing tensors at infinity ("improper gauge transformations"). They can be computed from the constraints [27] or equivalently from the knowledge of their associated conserved antisymmetric tensors $k_{\xi}^{[\alpha \beta]}$ introduced in [30], which generalize the electromagnetic $F_{\mu \nu}$, the divergence of which vanishes in the absence of sources. The corresponding charge is given by $Q_{\xi}=\frac{1}{2} \int_{S} \star k_{\xi}[\alpha \beta] d x^{\alpha} \wedge d x^{\beta}$, where the integral is taken at constant time, over the 2 -sphere at infinity. The tensors $k_{\xi}^{[\alpha \beta]} \mathrm{read}$

$$
\begin{aligned}
k_{\xi}^{[\alpha \beta]}= & \partial^{\alpha} h^{\beta \mu_{1} \cdots \mu_{s-1}} \xi_{\mu_{1} \cdots \mu_{s-1}}+\frac{(s-1)}{2} \partial^{\beta} h_{\rho}^{\rho \mu_{1} \cdots \mu_{s-2}} \xi_{\mu_{1} \cdots \mu_{s-2}}^{\alpha} \\
& +(s-1) \partial_{\rho} h^{\rho \alpha \mu_{1} \cdots \mu_{s-2}} \xi_{\mu_{1} \cdots \mu_{s-2}}^{\beta}-\frac{(s-1)^{2}}{2} \partial^{(\alpha} h_{\rho}^{{ }^{\left.\mu_{1} \cdots \mu_{s-2}\right) \rho}} \xi^{\beta}{ }_{\mu_{1} \cdots \mu_{s-2}} \\
& -(\alpha \leftrightarrow \beta)+\cdots,
\end{aligned}
$$

where the dots stand for terms involving derivatives of the gauge parameters.

Of particular interest are the charges corresponding to gauge transformations that are "asymptotic translations", i.e. $\xi^{\mu_{1} \cdots \mu_{s-1}} \rightarrow_{r \rightarrow \infty} \epsilon^{\mu_{1} \cdots \mu_{s-1}}$ for some traceless constant tensor $\epsilon^{\mu_{1} \cdots \mu_{s-1}}$. For these transformations, the charges become, using Stokes' theorem and the explicit expression for $k_{\xi}^{[\alpha \beta]}$,

$$
Q_{\epsilon}=\epsilon_{\mu_{1} \cdots \mu_{s-1}} \int_{V} G^{0 \mu_{1} \cdots \mu_{s-1}} d^{3} x .
$$

As these charges are conserved for any traceless $\epsilon_{\mu_{1} \cdots \mu_{s-1}}$, the quantities $P^{\mu_{1} \cdots \mu_{s-1}}$ defined as the traceless parts of $\int_{V} G^{0 \mu_{1} \cdots \mu_{s-1}} d^{3} x$ are conserved as well. In the spin 2 case, $P^{\mu}$ is the energy-momentum 4 -vector.

\section{Electric sources}

In the presence of only electric sources, the equations of motion read:

$$
G_{\mu_{1} \mu_{2} \cdots \mu_{s}}+T_{\mu_{1} \mu_{2} \cdots \mu_{s}}=0,
$$

or equivalently

$$
R_{\mu_{1} \nu_{1} \mu_{2} \nu_{2} \cdots \mu_{s} \nu_{s}} \eta^{\nu_{1} \nu_{2}}=\frac{1}{2} \bar{T}_{\mu_{1} \mu_{2}\left[\mu_{3}\left[\cdots\left[\mu_{s}, \nu_{s}\right] \cdots\right] \nu_{3}\right]}
$$


where $\bar{T}_{\mu_{1} \mu_{2} \cdots \mu_{s}}=T_{\mu_{1} \mu_{2} \cdots \mu_{s}}-\frac{s}{4} \eta_{\left(\mu_{1} \mu_{2}\right.} T_{\left.\mu_{3} \cdots \mu_{s}\right)}^{\prime}$ generalizes the energy momentum tensor and primes denote traces, $T_{\mu_{3} \cdots \mu_{s}}^{\prime}=T_{\mu_{1} \cdots \mu_{s}} \eta^{\mu_{1} \mu_{2}}$. The curvature tensor has the Young symmetry (A.3) and fulfills the Bianchi identity (A.4), as in the case without sources.

On the other hand, while the trace of the dual curvature tensor still vanishes, the latter has no longer the Young symmetry (A.3) and its Bianchi identity gets modified as well. The new symmetry is described by the Young tableau

$$
\begin{array}{|l|l|l|l|}
\hline \mu_{1} \\
\hline \nu_{1}
\end{array} \otimes \begin{array}{|l|l|l|}
\hline \mu_{2} & \cdots & \mu_{s} \\
\hline \nu_{2} & \cdots & \nu_{s} \\
\hline
\end{array}
$$

as the dual curvature now satisfies $S_{\left[\mu_{1} \nu_{1} \mu_{2}\right] \nu_{2} \cdots \mu_{s} \nu_{s}}=-\frac{1}{6} \epsilon_{\mu_{1} \nu_{1} \mu_{2} \rho} \bar{T}_{\nu_{2}\left[\mu_{3}\left[\cdots\left[\mu_{s}, \nu_{s}\right] \cdots\right] \nu_{3}\right]}^{\rho}$, while the Bianchi identity becomes $\partial_{[\alpha} S_{\left.\mu_{1} \nu_{1}\right] \mu_{2} \nu_{2} \cdots \mu_{s} \nu_{s}}=\frac{1}{3} \epsilon_{\alpha \mu_{1} \nu_{1} \rho} \bar{T}_{\left[\mu_{2}\left[\mu_{3}\left[\cdots\left[\mu_{s}, \nu_{s}\right] \cdots\right] \nu_{3}\right] \nu_{2}\right]}^{\rho}$.

\section{APPENDIX B: GAUGE INVARIANCES OF HIGHER SPIN ACTION (WITH MAGNETIC TERMS)}

We prove in this appendix that the action (III.22) is invariant both under the standard symmetries of the spin s gauge field and under displacements of the Dirac string.

To that end, we first observe that the first term in the action (III.22) is invariant under the following shifts of $Y^{\mu \nu}{ }_{\alpha_{1} \cdots \alpha_{s-1}}$ :

$$
\delta Y_{\alpha_{1} \cdots \alpha_{s-1}}^{\mu \nu}=\partial_{\rho} \delta_{\left(\alpha_{1}\right.}^{\mu} z_{\left.\alpha_{2} \cdots \alpha_{s-1}\right)}^{\nu \rho}-\partial_{\rho} \delta_{\left(\alpha_{1}\right.}^{\nu} z_{\left.\alpha_{2} \cdots \alpha_{s-1}\right)}^{\mu \rho}+\partial_{\left(\alpha_{1}\right.} z_{\left.\alpha_{2} \cdots \alpha_{s-1}\right)}^{\mu \nu}
$$

where $z^{\mu \nu}{ }_{\alpha_{1} \cdots \alpha_{s-2}}=z_{\alpha_{1} \cdots \alpha_{s-2}}^{[\mu \nu]}=z_{\left(\alpha_{1} \cdots \alpha_{s-2}\right)}^{\mu \nu}$ is an arbitrary traceless tensor that satisfies $\eta^{\alpha_{1}[\lambda} z^{\mu \nu]}{ }_{\alpha_{1} \cdots \alpha_{s-2}}=0$ when $s>2$. Under this transformation, $\bar{Y}^{\mu \nu}{ }_{{ }_{1} \cdots \alpha_{s-1}}$ transforms as $\delta \bar{Y}^{\mu \nu}{ }_{\alpha_{1} \cdots \alpha_{s-1}}=\partial_{\left(\alpha_{1}\right.} z_{\left.\alpha_{2} \cdots \alpha_{s-1}\right)}^{\mu \nu}$, which makes it obvious that the curvature and the Fronsdal tensor are invariant under (B.1).

The transformation (B.1) can be conveniently written

$$
\delta Y^{\mu \nu}{ }_{\alpha_{1} \cdots \alpha_{s-1}}=\epsilon^{\mu \nu \rho \sigma} \partial_{\rho} a_{\sigma \alpha_{1} \alpha_{2} \cdots \alpha_{s-1}},
$$

where $a_{\sigma \alpha_{1} \alpha_{2} \cdots \alpha_{s-1}}=-a_{\alpha_{1} \sigma \alpha_{2} \cdots \alpha_{s-1}}=a_{\sigma \alpha_{1}\left(\alpha_{2} \cdots \alpha_{s-1}\right)}$ is given by

$$
a_{\sigma \alpha_{1} \alpha_{2} \cdots \alpha_{s-1}}=\frac{1}{2} \epsilon_{\sigma \beta \gamma \alpha_{1}} z^{\beta \gamma}{ }_{\alpha_{2} \cdots \alpha_{s-1}},
$$

is traceless and satisfies $a_{\left[\sigma \alpha_{1} \alpha_{2}\right] \alpha_{3} \cdots \alpha_{s-1}}=0$ when $s>2$.

Direct computation shows that the gauge transformation (A.1) of the spin s field acts on $Y^{\mu \nu}{ }_{\alpha_{1} \cdots \alpha_{s-1}}$ as the transformation (B.2) with

$$
\begin{aligned}
a_{\rho \sigma\left(\alpha_{1} \cdots \alpha_{s-2}\right)}= & -2 \frac{(s-1)}{s} \partial_{[\rho} \xi_{\sigma] \alpha_{1} \cdots \alpha_{s-2}} \\
& +\frac{(s-1)(s-2)}{s^{2}}\left[\eta_{\rho\left(\alpha_{1}\right.} \partial^{\lambda} \xi_{\left.\alpha_{2} \cdots \alpha_{s-2}\right) \lambda \sigma}-\eta_{\sigma\left(\alpha_{1}\right.} \partial^{\lambda} \xi_{\left.\alpha_{2} \cdots \alpha_{s-2}\right) \lambda \rho}\right] .
\end{aligned}
$$

It follows from this fact and the conservation of the energy-momentum tensor that the action (III.22) is invariant under the standard gauge transformation (A.1) of the spin s field.

The displacements of the Dirac string change $\Phi^{\mu \nu}{ }_{\alpha_{1} \cdots \alpha_{s-1}}$ as $\delta \Phi^{\mu \nu}{ }_{\alpha_{1} \cdots \alpha_{s-1}}=k^{\mu \nu}{ }_{\alpha_{1} \cdots \alpha_{s-1}}$ where $\partial_{\mu} k^{\mu \nu}{ }_{\alpha_{1} \cdots \alpha_{s-1}}=$ 0 . The latter equation implies that $k_{\alpha_{1} \cdots \alpha_{s-1}}^{\mu \nu}=\partial_{\lambda} K_{\alpha_{1} \cdots \alpha_{s-1}}^{\mu \nu \lambda}$, where $K_{\alpha_{1} \cdots \alpha_{s-1}}^{\mu \nu \lambda}=K_{\alpha_{1} \cdots \alpha_{s-1}}^{[\mu \nu \lambda]}$. Let $\hat{K}_{\alpha_{1} \cdots \alpha_{s-1}}^{\mu \nu \lambda}$ be the part of $K_{\alpha_{1} \cdots \alpha_{s-1}}^{\mu \nu \lambda}$ that is traceless in $\alpha_{1} \cdots \alpha_{s-1}$; it can be decomposed as

$$
\hat{K}_{\alpha_{1} \cdots \alpha_{s-1}}^{\mu \nu \lambda}=x_{\alpha_{1} \cdots \alpha_{s-1}}^{\mu \nu \lambda}+\delta_{\left(\alpha_{1}\right.}^{[\lambda} y_{\left.\alpha_{2} \cdots \alpha_{s-1}\right)}^{\mu \nu]},
$$

where $x^{\mu \nu \lambda}{ }_{\alpha_{1} \cdots \alpha_{s-1}}$ and $y^{\mu \nu}{ }_{\alpha_{2} \cdots \alpha_{s-1}}$ satisfy

$$
\begin{aligned}
& x_{\alpha_{1} \cdots \alpha_{s-1}}^{\mu \nu \lambda}=x_{\alpha_{1} \cdots \alpha_{s-1}}^{[\mu \nu \lambda]}=x_{\left(\alpha_{1} \cdots \alpha_{s-1}\right)}^{\mu \nu \lambda}, x^{\mu \nu \lambda}{ }_{\alpha_{1} \cdots \alpha_{s-1}} \delta_{\lambda}^{\alpha_{1}}=0 \text {, } \\
& y_{\alpha_{2} \cdots \alpha_{s-1}}^{\mu \nu}=y_{\alpha_{2} \cdots \alpha_{s-1}}^{[\mu \nu]}=y_{\left(\alpha_{2} \cdots \alpha_{s-1}\right)}^{\mu \nu}, y_{\alpha_{2} \cdots \alpha_{s-1}}^{\mu \nu} \delta_{\nu}^{\alpha_{1}}=0, \\
& \eta^{\alpha_{1}[\lambda} y_{\alpha_{1} \cdots \alpha_{s-1}}^{\mu \nu]}=-\frac{(s-1)}{2} x_{\alpha_{1} \cdots \alpha_{s-1}}^{\mu \nu \lambda} \eta^{\alpha_{1} \alpha_{2}}, y^{\mu \nu}{ }_{\alpha_{1} \cdots \alpha_{s-1}} \eta^{\alpha_{1} \alpha_{2}}=0 .
\end{aligned}
$$


For the action to be invariant under displacements of the string, the variation of $\Phi^{\mu \nu}{ }_{\alpha_{1} \cdots \alpha_{s-1}}$ has to be supplemented with an appropriate transformation of $h_{\alpha_{1} \cdots \alpha_{s}}$. This transformation reads $\delta h_{\alpha_{1} \cdots \alpha_{s}}=\frac{1}{6} \epsilon_{\mu \nu \lambda\left(\alpha_{1}\right.} x_{\left.\alpha_{2} \cdots \alpha_{s}\right)}^{\mu \nu \lambda}$. Indeed, when one performs both variations, $Y^{\mu \nu}{ }_{\alpha_{1} \cdots \alpha_{s-1}}$ transforms as in (B.1), so the first term in the action is invariant. Furthermore, the electric coupling term is invariant as well because the support of the variation of the spin s field does not contain the electric worldlines.

The identities that follow from the invariance (B.1) - or (B.2) - of the first term $\mathcal{L}$ in the action may be written conveniently in terms of

$$
A^{\sigma \gamma_{1} \cdots \gamma_{s-1}}=\epsilon^{\sigma \mu \nu \lambda} \partial_{\lambda}\left(\frac{\partial \mathcal{L}}{\partial Y^{\mu \nu} \gamma_{1} \cdots \gamma_{s-1}}\right)
$$

and its trace $A^{\prime \gamma_{2} \cdots \gamma_{s-1}}=A^{\sigma \gamma_{1} \cdots \gamma_{s-1}} \eta_{\sigma \gamma_{1}}$. They read

$$
0=A^{\sigma \gamma_{1} \cdots \gamma_{s-1}}-A^{\left(\gamma_{1} \gamma_{2} \cdots \gamma_{s-1}\right) \sigma}-\frac{s-2}{s}\left(\eta^{\sigma\left(\gamma_{1}\right.} A^{\left.\prime \gamma_{2} \cdots \gamma_{s-1}\right)}-\eta^{\left(\gamma_{1} \gamma_{2}\right.} A^{\left.\prime \gamma_{3} \cdots \gamma_{s-1}\right) \sigma}\right) .
$$

Using these identities, one checks the following useful relations:

$$
\begin{aligned}
G^{\gamma_{1} \cdots \gamma_{s}}=\frac{\delta \mathcal{L}}{\delta h_{\gamma_{1} \cdots \gamma_{s}}} & =A^{\left(\gamma_{1} \gamma_{2} \cdots \gamma_{s}\right)}+\frac{(s-1)(s-2)}{2 s} \eta^{\left(\gamma_{1} \gamma_{2}\right.} A^{\left.\prime \gamma_{3} \cdots \gamma_{s}\right)} \\
& =A^{\gamma_{1} \gamma_{2} \cdots \gamma_{s}}+\frac{(s-1)(s-2)}{2 s} \eta^{\left(\gamma_{2} \gamma_{3}\right.} A^{\left.\prime \gamma_{4} \cdots \gamma_{s}\right) \gamma_{1}}
\end{aligned}
$$

which will be used in the following appendix.

\section{APPENDIX C: QUANTIZATION CONDITION FOR ELECTRIC AND MAGNETIC HIGHER SPIN SOURCES}

We work out explicitly in this appendix the quantization condition for higher spins. As for lower spins, this is achieved by integrating the constraints expressing the unobservability of the Dirac string.

In the gauge $y^{0}=\lambda$, these constraints read

$$
\pi_{m}=-2 N y^{\prime n} f_{\gamma_{1} \cdots \gamma_{s-1}}(v) \frac{\partial \mathcal{L}}{\partial Y^{m n}{ }_{\gamma_{1} \cdots \gamma_{s-1}}} .
$$

In the quantum theory, the wave functional $\psi$ must thus fulfill

$$
\frac{\hbar}{i} \frac{\delta \psi}{\delta y^{m}(\sigma)}=-2 N y^{\prime n} f_{\gamma_{1} \cdots \gamma_{s-1}}(v) \frac{\partial \mathcal{L}}{\partial Y^{m n}{ }_{\gamma_{1} \cdots \gamma_{s-1}}} \psi .
$$

Integrating this equation along a path that encloses an electric source, one finds the following variation of the phase of the wave functional

$$
\Delta \Psi=-\frac{N}{\hbar} f_{\gamma_{1} \cdots \gamma_{s-1}}(v) \int \frac{\partial \mathcal{L}}{\partial Y^{m n}{ }_{\gamma_{1} \cdots \gamma_{s-1}}}\left(\dot{y}^{m} y^{\prime n}-\dot{y}^{n} y^{\prime m}\right) d \sigma d \lambda,
$$

where the integral is taken on the two-dimensional surface enclosing the electric source. Using the Gauss theorem, this can be converted into a volume integral,

$$
\Delta \Psi=-\frac{N}{\hbar} f_{\gamma_{1} \cdots \gamma_{s-1}}(v) \int d^{3} x \epsilon^{m n p} \partial_{p}\left(\frac{\partial \mathcal{L}}{\partial Y^{m n}{ }_{\gamma_{1} \cdots \gamma_{s-1}}}\right) .
$$

Using the relations (B.7), one checks that

$$
\epsilon^{m n p} \partial_{p}\left(\frac{\partial \mathcal{L}}{\partial Y^{m n} \gamma_{1} \cdots \gamma_{s-1}}\right)=\frac{\delta \mathcal{L}}{\delta h_{0 \gamma_{1} \cdots \gamma_{s-1}}}+\cdots,
$$


where the dots stand for terms of the form $\eta^{\left(\gamma_{1} \gamma_{2}\right.} X^{\left.\gamma_{3} \cdots \gamma_{s-1}\right)}$. Upon use of the Einstein equations $G^{0 \gamma_{1} \cdots \gamma_{s-1}}=$ $-T^{0 \gamma_{1} \cdots \gamma_{s-1}}$, the variation of the phase becomes, $\Delta \Psi=\frac{N}{\hbar} f_{\gamma_{1} \cdots \gamma_{s-1}}(v) \int d^{3} x T^{0 \gamma_{1} \cdots \gamma_{s-1}}=\frac{M N}{\hbar} f_{\gamma_{1} \cdots \gamma_{s-1}}(v) f^{\gamma_{1} \cdots \gamma_{s-1}}(u)$. For the wave functional to be single-valued, this should be a multiple of $2 \pi$. This yields the quantization condition

$$
\frac{M N}{2 \pi \hbar} f_{\gamma_{1} \cdots \gamma_{s-1}}(v) f^{\gamma_{1} \cdots \gamma_{s-1}}(u)=n, \quad n \in \mathbb{Z} .
$$

Introducing the conserved charges $P^{\gamma_{1} \cdots \gamma_{s-1}}, Q^{\gamma_{1} \cdots \gamma_{s-1}}$, this can be rewritten as

$$
\frac{1}{2 \pi \hbar} Q_{\gamma_{1} \cdots \gamma_{s-1}}(v) P^{\gamma_{1} \cdots \gamma_{s-1}}(u) \in \mathbb{Z}
$$

\section{APPENDIX D: ROTATION SYMMETRY AND CHARGE QUANTIZATION FOR AN ELECTROMAGNETIC MAGNETIC POLE}

\section{The Dirac string and rotations}

A monopole is spherically symmetric, yet the vector potential is not manifestly so, and when rotated, the string will move to a new location. As is well known, spherical symmetry is recovered by supplementing the naive rotation generated by the usual Killing vectors by a gauge transformation that moves the string back to its original location. Let us see how this works explicitly.

The usual Killing vectors which generate $\mathrm{SO}(3)$ rotations on the two-sphere are given by

$$
\begin{aligned}
\xi_{X} & =-\sin \phi \frac{\partial}{\partial \theta}-\cos \phi \cot \theta \frac{\partial}{\partial \phi}, \\
\xi_{Y} & =\cos \phi \frac{\partial}{\partial \theta}-\sin \phi \cot \theta \frac{\partial}{\partial \phi} \\
\xi_{Z} & =\frac{\partial}{\partial \phi} .
\end{aligned}
$$

They satisfy the algebra

$$
\left[\xi_{A}, \xi_{B}\right]=-\epsilon_{A B C} \xi_{C}
$$

Under an infinitesimal rotation generated by one of these vectors, the vector potential changes by the Lie derivative along the vector field:

$$
\mathcal{L}_{\xi_{B}} A_{i}=\xi_{B}^{j} \partial_{j} A_{i}+A_{j} \partial_{i} \xi_{B}^{j}=\xi_{B}^{j} F_{j i}+\partial_{i}\left(\xi_{B}^{j} A_{j}\right) .
$$

For the monopole vector potential (D.11) this Lie derivative is non-zero and hence usual rotational symmetry would appear to be broken. However, we can undo its effect if we accompany the Lie derivative by a gauge transformation $A_{i} \rightarrow A_{i}+\partial_{i} \Lambda$ such that the total change is

$$
\delta_{\xi_{B}} A_{i}=\mathcal{L}_{\xi_{B}} A_{i}+\partial_{i} \Lambda_{B}=0 .
$$

The gauge transformations that we find associated with the three Killing vector fields are

$$
\begin{aligned}
& \Lambda_{X}=-\frac{g}{4 \pi} \cos \phi \sin \theta-\xi_{X}^{j} A_{j}=\frac{g k}{4 \pi} \cot \theta \cos \phi-\frac{g}{4 \pi} \frac{\cos \phi}{\sin \theta} \\
& \Lambda_{Y}=-\frac{g}{4 \pi} \sin \phi \sin \theta-\xi_{Y}^{j} A_{j}=\frac{g k}{4 \pi} \cot \theta \sin \phi-\frac{g}{4 \pi} \frac{\sin \phi}{\sin \theta} \\
& \Lambda_{Z}=-\frac{g}{4 \pi} \cos \theta-\xi_{Z}^{j} A_{j}=-\frac{g k}{4 \pi} .
\end{aligned}
$$

We note that $\Lambda_{Z}$ is a constant gauge parameter, yet it needs to be considered in order that the new rotation generators to be defined below satisfy the algebra.

We can combine the gauge transformations $\Lambda_{B}$ and the naive rotation operators $\xi_{B}$ to create new rotation operators $\hat{\xi}_{B}=\xi_{B}+\Lambda_{B} \frac{\partial}{\partial \lambda}$ by using the fact that the generator of gauge transformations is the electric charge $q$, which is in 
turn conjugate to the coordinate on the $\mathrm{U}(1)$ fiber which we call $\lambda$, so we can write:

$$
\begin{aligned}
& \hat{\xi}_{X}=-\sin \phi \frac{\partial}{\partial \theta}-\cos \phi \cot \theta \frac{\partial}{\partial \phi}+\left(\frac{g k}{4 \pi} \cos \phi \cot \theta-\frac{g}{4 \pi} \frac{\cos \phi}{\sin \theta}\right) \frac{\partial}{\partial \lambda} \\
& \hat{\xi}_{Y}=\cos \phi \frac{\partial}{\partial \theta}-\sin \phi \cot \theta \frac{\partial}{\partial \phi}+\left(\frac{g k}{4 \pi} \sin \phi \cot \theta-\frac{g}{4 \pi} \frac{\sin \phi}{\sin \theta}\right) \frac{\partial}{\partial \lambda} \\
& \hat{\xi}_{Z}=\frac{\partial}{\partial \phi}-\frac{g k}{4 \pi} \frac{\partial}{\partial \lambda}
\end{aligned}
$$

These operators satisfy the $\mathrm{SO}(3)$ algebra just as (D.1).

\section{The field angular momentum}

Let us now consider an electric charge $q$ of mass $m$ placed at a point $\vec{a}$ in the background field of a magnetic pole $g$ placed at the origin. The background magnetic and dynamical electric fields are

$$
\vec{B}=\frac{g \vec{x}}{4 \pi|x|^{3}}, \quad \vec{E}=\frac{q(\vec{x}-\vec{a})}{4 \pi|x-a|^{3}} .
$$

As is well known, the angular momentum stored in the electromagnetic field does not vanish. It can be calculated from the standard symmetric energy-momentum tensor of the electromagnetic field and is given by

$$
\vec{L}_{\text {field }}=\int_{V} \vec{x} \times(\vec{E} \times \vec{B})=\frac{q g}{4 \pi} \frac{\vec{a}}{|a|} .
$$

This expression is independent of the magnitude of $\vec{a}$, though it depends on its direction, and therefore the limit $\vec{a} \rightarrow 0$ cannot be taken continuously. For example, if we place the charge above the pole the angular momentum will be negative, whereas it will be positive if placed below. Whatever the sign, if we assume the angular momentum to be quantized in integer multiples of $\hbar / 2$, we obtain the quantization condition

$$
\frac{q g}{2 \pi} \in \mathbb{Z}
$$

This was first noted by Fierz and is the statement that quantization of the field angular momentum results in the Dirac quantization condition. The fact that one may obtain half integer angular momentum without fermions in sight has been lucidly analyzed in [31].

\section{Angular momentum of a test electric charge in the background of a magnetic monopole}

One can get direct access to the angular momentum in the field differently, by analyzing the angular momentum of a test electric charge in the background of a magnetic pole.

The magnetic field of a magnetic monopole of strength $g$,

$$
B^{r}=\frac{g}{4 \pi r^{2}}
$$

can be associated with the one form vector potential

$$
A=\frac{g}{4 \pi}(k-\cos \theta) d \phi
$$

which satisfies $B=\nabla \times A$, for $k$ an arbitrary constant, away from the Dirac string. When $k=1$ the Dirac string lies along the negative $z$-axis while when $k=-1$ the Dirac string lies along the positive $z$-axis. The value of $k$ can be modified to $\tilde{k}$ by a gauge transformation with parameter $g(\tilde{k}-k) \phi / 4 \pi$. For values of $k \neq \pm 1$, there are two strings, one along the positive $z$-axis and one along the negative $z$-axis, with strengths that add up to one.

By demanding that the velocity should transform as a vector under rotations, the angular momentum operator

$$
\vec{J}=m \vec{x} \times \dot{\vec{x}}+\frac{q g}{4 \pi} \frac{\vec{x}}{|x|},
$$


was derived in [32].

There is an alternative way to arrive at this result which we believe is worth recalling. Using the standard Noether procedure for the action

$$
S=\int\left(\frac{1}{2} m \dot{x}_{i} \dot{x}^{i}-q A_{i} \dot{x}^{i}\right) d t
$$

we obtain that for a variation $\delta x$ the action changes by

$$
\delta S=\int \frac{d}{d t}\left[\left(m \dot{x}_{i}-q A_{i}\right) \delta x^{i}\right]
$$

on-shell, i.e. when the equations of motion hold. The transformation will generate a conserved charge if the action is invariant off-shell up to the integral of a total derivative. Let us consider what the case is for a rotation generated by $\delta x^{i}=\xi_{B}^{i}$. The first term in (D.13) is the norm of a vector and therefore manifestly invariant under rotations. The variation of the second term becomes

$$
\begin{aligned}
\delta S & =-q \int\left(\dot{x}^{i} \delta_{\xi_{B}} A_{i}+A_{i} \delta_{\xi_{B}} \dot{x}^{i}\right) \\
& =-q \int\left(\mathcal{L}_{\xi_{B}} A_{i}\right) \dot{x}^{i}
\end{aligned}
$$

where we have used $\delta_{\xi_{B}} A_{i}=\left(\partial_{j} A_{i}\right) \delta_{\xi_{B}} x^{j}$. If, as is the case here (see (D.4)), the Lie derivative of $A_{i}$ is given by a total derivative $\mathcal{L}_{\xi_{B}} A_{i}=-\partial_{i} \Lambda_{B}$ this becomes

$$
\delta S=q \int \frac{d}{d t}\left[\Lambda_{B}\right] .
$$

We can now equate equations (D.14) and (D.16) to obtain the charge $J_{B}$ conserved under rotations

$$
J_{B}=\left(m \dot{x}_{i}-q A_{i}\right) \xi_{B}^{i}-q \Lambda_{B}
$$

In fact the $\Lambda_{B}$ are the gauge transformations we computed in (D.5). For example, for rotations about the z-axis, we obtain that

$$
J_{Z}=m \dot{\phi}+\frac{q g}{4 \pi} \cos \theta
$$

and in general the result (D.12).

From the expression (D.12), it is clear that the angular momentum does not vanish when the particle is at rest. This apparent paradox is explained by comparing (D.12) with (D.8). The extra piece is the angular momentum of the electromagnetic field, which must be included because the electric field of the test particle is dynamical and hence, the angular momentum of the electromagnetic field changes with time. Only the sum of the standard orbital angular momentum and the field angular momentum is conserved.

Expressing the angular momentum in terms of the (non gauge invariant) conjugate momenta $p_{\phi}=m \dot{\phi}-q A_{\phi}$ one obtains, for the angular momentum about the z-axis,

$$
J_{Z}=p_{\phi}+k \frac{q g}{4 \pi}
$$

In the gauge $k=1$ and when the particle is on the positive $z$-axis, the field angular momentum is just equal to the extra piece appearing in $J_{Z}$, besides the usual $p_{\phi}$. This is a way to identify the field angular momentum if one knows the total angular momentum, as it is the case for gravity. Note that in the gauge $k=0$, $p_{\phi}$ is equal to the total angular momentum.

It has been shown in $[31,32]$, that the difference $J_{Z}-q g / 4 \pi$ has integer eigenvalues. We may rewrite this assertion in the notation used here as the statement that in the gauge $k=1$ the operator $p_{\phi}=\frac{\hbar}{i} \frac{\partial}{\partial \phi}$ has integer eigenvalues and therefore the wave function is periodic in $\phi$.

However the periodicity of the wave function depends on $k$. This can be traced to the fact that differentiating with respect to $\phi$

$$
p_{\phi}=\left.\frac{\hbar}{i} \frac{\partial}{\partial \phi}\right|_{\lambda}
$$


at constant $\lambda$ (where $\lambda$ is the coordinate along the $\mathrm{U}(1)$ fiber) is not invariant under the gauge transformation

$$
\lambda \rightarrow \lambda-\frac{g \phi}{4 \pi}
$$

which brings $k$ from 1 to 0 . As (D.21) itself shows the wave function picks up a phase factor $e^{-i \frac{q g}{4 \pi} \phi}$ which shows in turn, that in the gauge $k=0$ the wave function is antiperiodic in $\phi$ when $q g / 4 \pi$ is half integer. This anti-periodicity is permissible because in the gauge $k=0$ an infinite line is removed from $R^{3}$ and the resulting configuration space for the electron is therefore not simply connected. The infinite line is formed by two strings of half strength that come into the magnetic pole along the positive and negative sides of the $z$-axis.

There is another interesting manifestation of the lack of simple connectedness due to the infinite line formed by the two strings when $k=0$. It is the following: the closed path traveled in configuration space when the string sweeps out a closed surface around the electric charge is not contractible to the identity, whereas it is so when the string is half infinite (see [10]). For this reason the string wave function changes sign after the turn and one gets the same quantization condition as when $k=1$ in which case the path is contractible and the wave function returns to its original value.

[1] P. A. M. Dirac, "Quantised Singularities In The Electromagnetic Field," Proc. Roy. Soc. Lond. A 133 (1931) 60.

[2] P. A. M. Dirac, "The Theory Of Magnetic Poles," Phys. Rev. 74, 817 (1948).

[3] G. 't Hooft, "Magnetic Monopoles In Unified Gauge Theories," Nucl. Phys. B 79 (1974) 276.

[4] A. M. Polyakov, "Particle Spectrum In Quantum Field Theory," JETP Lett. 20 (1974) 194 [Pisma Zh. Eksp. Teor. Fiz. 20 (1974) 430].

[5] C. Montonen and D. I. Olive, "Magnetic Monopoles As Gauge Particles?," Phys. Lett. B 72 (1977) 117.

[6] C. Teitelboim, "Gauge Invariance For Extended Objects," Phys. Lett. B 167, 63 (1986);

C. Teitelboim, "Monopoles Of Higher Rank," Phys. Lett. B 167, 69 (1986).

[7] R. I. Nepomechie, "Magnetic Monopoles From Antisymmetric Tensor Gauge Fields," Phys. Rev. D 31, 1921 (1985).

[8] J. S. Schwinger, "Sources And Magnetic Charge," Phys. Rev. 173, 1536 (1968); J. S. Schwinger, "A Magnetic Model Of Matter," Science 165 (1969) 757.

[9] D. Zwanziger, "Quantum Field Theory Of Particles With Both Electric And Magnetic Charges," Phys. Rev. 176, 1489 (1968).

[10] S. Deser, A. Gomberoff, M. Henneaux and C. Teitelboim, "Duality, self-duality, sources and charge quantization in abelian N-form theories," Phys. Lett. B 400, 80 (1997) [arXiv:hep-th/9702184];

S. Deser, A. Gomberoff, M. Henneaux and C. Teitelboim, "p-brane dyons and electric-magnetic duality," Nucl. Phys. B 520, 179 (1998) [arXiv:hep-th/9712189].

[11] C. Fronsdal, "Massless Fields With Integer Spin," Phys. Rev. D 18 (1978) 3624.

[12] B. de Wit and D. Z. Freedman, "Systematics Of Higher Spin Gauge Fields," Phys. Rev. D 21, 358 (1980);

T. Curtright, "Generalized Gauge Fields," Phys. Lett. B 165, 304 (1985).

[13] H. Garcia-Compean, O. Obregon and C. Ramirez, "Gravitational duality in MacDowell-Mansouri gauge theory," Phys. Rev. D 58, 104012 (1998) [arXiv:hep-th/9802063];

J. A. Nieto, "S-duality for linearized gravity," Phys. Lett. A 262, 274 (1999) [arXiv:hep-th/9910049];

C. M. Hull, "Duality in gravity and higher spin gauge fields," JHEP 0109, 027 (2001) [arXiv:hep-th/0107149];

D. Francia and A. Sagnotti, "Free geometric equations for higher spins," Phys. Lett. B 543, 303 (2002) [arXiv:hepth/0207002];

X. Bekaert and N. Boulanger, "Tensor gauge fields in arbitrary representations of GL(D,R): Duality and Poincare lemma," Commun. Math. Phys. 245, 27 (2004) [arXiv:hep-th/0208058];

P. de Medeiros and C. Hull, "Geometric second order field equations for general tensor gauge fields," JHEP 0305, 019 (2003) [arXiv:hep-th/0303036];

H. Casini, R. Montemayor and L. F. Urrutia, "Duality for symmetric second rank tensors. II: The linearized gravitational field," Phys. Rev. D 68, 065011 (2003) [arXiv:hep-th/0304228];

N. Bouatta, G. Compere and A. Sagnotti, "An introduction to free higher-spin fields," arXiv:hep-th/0409068;

Y. M. Zinoviev, "On dual formulation of gravity," arXiv:hep-th/0504210;

Y. M. Zinoviev, "On dual formulation of gravity. II: Metric and affine connection," arXiv:hep-th/0506217;

D. Francia and A. Sagnotti, "Minimal local Lagrangians for higher-spin geometry," Phys. Lett. B 624, 93 (2005) [arXiv:hepth/0507144].

[14] D. Francia and A. Sagnotti, "On the geometry of higher-spin gauge fields, Class. Quant. Grav. 20, S473 (2003) [arXiv:hepth/0212185]. 
[15] X. Bekaert and N. Boulanger, "On geometric equations and duality for free higher spins," Phys. Lett. B 561, 183 (2003) [arXiv:hep-th/0301243].

[16] N. Boulanger, S. Cnockaert and M. Henneaux, "A note on spin-s duality," JHEP 0306, 060 (2003) [arXiv:hep-th/0306023].

[17] M. Henneaux and C. Teitelboim, "Duality in linearized gravity," Phys. Rev. D 71 (2005) 024018 [arXiv:gr-qc/0408101].

[18] B. Julia, J. Levie and S. Ray, "Gravitational duality near de Sitter space," JHEP 0511, 025 (2005)[arXiv:hep-th/0507262]; B. L. Julia, "Electric-magnetic duality beyond four dimensions and in general relativity," arXiv:hep-th/0512320.

[19] M. A. Vasiliev, "Consistent Equation For Interacting Gauge Fields Of All Spins In (3+1)-Dimensions," Phys. Lett. B 243, 378 (1990);

M. A. Vasiliev, "More on equations of motion for interacting massless fields of all spins in (3+1)-dimensions," Phys. Lett. B 285, 225 (1992);

M. A. Vasiliev, "Nonlinear equations for symmetric massless higher spin fields in (A)dS(d)," Phys. Lett. B 567 (2003) 139 [arXiv:hep-th/0304049];

For a review, see X. Bekaert, S. Cnockaert, C. Iazeolla and M. A. Vasiliev, "Non linear higher spin theories in various dimensions," arXiv:hep-th/0503128, Proceedings of the 1st Solvay Workshop on Higher Spin Gauge Fields (Brussels, May 2004).

[20] E. Newman, L. Tamburino and T. Unti, "Empty Space Generalization Of The Schwarzschild Metric," J. Math. Phys. 4, 915 (1963).

[21] S. W. MacDowell and F. Mansouri, "Unified Geometric Theory Of Gravity And Supergravity," Phys. Rev. Lett. 38, 739 (1977) [Erratum-ibid. 38, 1376 (1977)].

[22] P. Olver, "Differential Hyperforms I", University of Minnesota report 82-101;

M. Dubois-Violette and M. Henneaux, "Generalized cohomology for irreducible tensor fields of mixed Young symmetry type," Lett. Math. Phys. 49, 245 (1999) [arXiv:math.qa/9907135];

M. Dubois-Violette and M. Henneaux, "Tensor fields of mixed Young symmetry type and N-complexes," Commun. Math. Phys. 226, 393 (2002) [arXiv:math.qa/0110088].

[23] S. Deser and D. Seminara, "Free spin 2 duality invariance cannot be extended to GR," Phys. Rev. D 71 (2005) 081502 [arXiv:hep-th/0503030].

[24] J. Ehlers, in Les théories relativistes de la gravitation (CNRS, Paris, 1959);

R. Geroch, J. Math. Phys. 12, 918 (1971);

B. Julia, LPTENS 80/16 - Invited paper presented at Nuffield Gravity Workshop, Cambridge, Jun 22 - Jul 12 , 1980.

[25] A. Zee, "Gravitomagnetic Pole And Mass Quantization," Phys. Rev. Lett. 55, 2379 (1985) [Erratum-ibid. 56, 1101 (1986)]; S. Ramaswamy and A. Sen, "Comment On 'Gravitomagnetic Pole And Mass Quantization.'," Phys. Rev. Lett. 57, 1088 (1986);

J. Samuel and B. R. Iyer, "Comment On 'Gravitomagnetic Pole And Mass Quantization.'," Phys. Rev. Lett. 57, 1089 (1986);

M. T. Mueller and M. J. Perry, "Constraints On Magnetic Mass," Class. Quant. Grav. 3, 65 (1986);

A. Magnon, "Global Techniques, Dual Mass, And Causality Violation," J. Math. Phys. 27, 1066 (1986).

[26] C. W. Misner, "The Flatter Regions of Newman, Unti, and Tamburino's Generalized Schwarzschild Space," Journal of Mathematical Physics 4 No.7 (1963) 924.

[27] T. Regge and C. Teitelboim, "Role Of Surface Integrals In The Hamiltonian Formulation Of General Relativity," Annals Phys. 88, 286 (1974).

[28] M. Demiański and E.T. Newman , "A Combined Kerr-NUT Solution of the Einstein Field Equations," Bull. Acad. Pol. Sc. 14, 653 (1966).

[29] S. Ramaswamy and A. Sen, "Dual-mass in general relativity," J. Math. Phys. 22, 2612 (1981);

A. Ashtekar and A. Sen, "NUT 4-momenta are forever," J. Math. Phys. 23, 2168 (1982).

[30] G. Barnich, N. Bouatta and M. Grigoriev, "Surface charges and dynamical Killing tensors for higher spin gauge fields in constant curvature spaces," arXiv:hep-th/0507138.

[31] A. S. Goldhaber, "Spin And Statistics Connection For Charge - Monopole Composites," Phys. Rev. Lett. 36 (1976) 1122.

[32] S. R. Coleman, "The Magnetic Monopole Fifty Years Later," HUTP-82/A032 Lectures given at Int. Sch. of Subnuclear Phys., Erice, Italy, Jul 31-Aug 11, 1981, at 6th Brazilian Symp. on Theor. Phys., Jan 7-18, 1980, at Summer School in Theoretical Physics, Les Houches, France, and at Banff Summer Inst. on Particle E Fields, Aug 16-28, 1981

[33] In terms of the Riemann tensor, this "identity" is a non trivial equation and not an identity. It becomes an identity only after the Riemann tensor is expressed in terms of the spin 2 field $h_{\mu \nu}$ introduced below. We shall nevertheless loosely refer to this equation as the (generalized) cyclic identity. A similar remark holds for the Bianchi identity below.

[34] We have been kindly informed by Donald Marolf that he can also vary the magnetic mass $N$ in a covariant formalism that he has developed. 